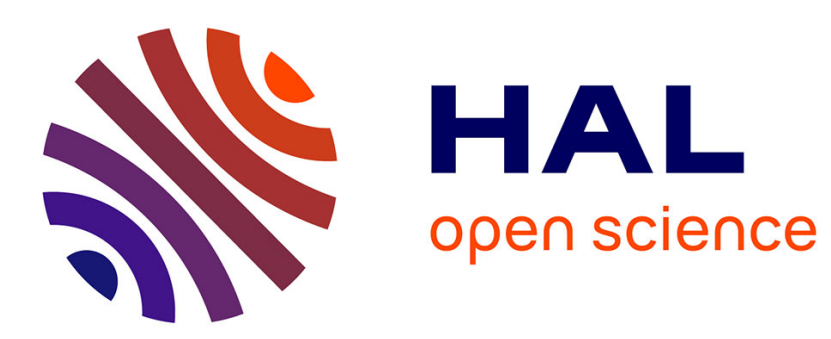

\title{
Optimal monodomain approximations of the bidomain equations used in cardiac electrophysiology
}

Yves Coudière, Yves Bourgault, Myriam Rioux

\section{To cite this version:}

Yves Coudière, Yves Bourgault, Myriam Rioux. Optimal monodomain approximations of the bidomain equations used in cardiac electrophysiology. Mathematical Models and Methods in Applied Sciences, 2014, 24 (6), pp.1115-1140. 10.1142/S0218202513500784 . hal-00644257

\section{HAL Id: hal-00644257 \\ https://hal.inria.fr/hal-00644257}

Submitted on 23 Nov 2011

HAL is a multi-disciplinary open access archive for the deposit and dissemination of scientific research documents, whether they are published or not. The documents may come from teaching and research institutions in France or abroad, or from public or private research centers.
L'archive ouverte pluridisciplinaire HAL, est destinée au dépôt et à la diffusion de documents scientifiques de niveau recherche, publiés ou non, émanant des établissements d'enseignement et de recherche français ou étrangers, des laboratoires publics ou privés. 
Optimal monodomain approximations of the bidomain equations used in cardiac electrophysiology

Yves Coudière, Yves Bourgault, Myriam Rioux

RESEARCH

REPORT

$\mathrm{N}^{\circ} \mathbf{7 8 1 0}$

November 2011

Project-Team Carmen 



\title{
Inzián
}

\section{Optimal monodomain approximations of the bidomain equations used in cardiac electrophysiology}

\author{
Yves Coudière* $\dagger$, Yves Bourgault ${ }^{\ddagger}$, Myriam Rioux ${ }^{\ddagger}$ \\ Project-Team Carmen \\ Research Report $\mathrm{n}^{\circ} 7810$ - November $2011-28$ pages
}

\begin{abstract}
The bidomain model is the current most sophisticated model used in cardiac electrophysiology. The monodomain model is a simplification of the bidomain model that is less computationally intensive but only valid under equal conductivity ratio. We propose in this paper optimal monodomain approximations of the bidomain model. We first prove that the error between the bidomain and monodomain solutions is bounded by the error $\|B-A\|$ between the bidomain and monodomain conductivity operators. Optimal monodomain approximations are defined by minimizing the distance $\|B-A\|$, which reduces for solutions over all $\mathbb{R}^{d}$ to minimize the $L^{p}$ norm of the difference between the operator symbols. Similarly, comparing the symbols pointwise amounts to compare the propagation of planar waves in the bidomain and monodomain models. We prove that any monodomain model properly propagates at least $d$ planar waves in $\mathbb{R}^{d}$. We next consider and solve the optimal problem in the $L^{\infty}$ and $L 2$ norms, the former providing minimal propagation error uniformly over all directions. The quality of these optimal monodomain approximations is compared among themselves and with other published approximations using two sets of test cases. The first one uses periodic boundary conditions to mimic propagation in $\mathbb{R}^{d}$ while the second is based on a square domain with common Neumann boundary conditions. For the first test cases, we show that the error on the propagation speed is highly correlated with the error on the symbols. The second test cases show that domain boundaries control propagation directions, with only partial impact from the conductivity operator used.
\end{abstract}

Key-words: Bidomain, monodomain, electrocardiology

* INRIA, 351 cours de la libération, 33400 Talence, France

$\dagger$ LMJL UMR6629 CNRS - Université de Nantes, 44300 Nantes, France

$¥$ Department of Mathematics and Statistics, University of Ottawa, 585 King Edward Av., Ottawa (Ont), Canada K1N 6N5 


\section{Approximations optimales de type monodomaine des équations du modèle bidomaine de l'électrophysiologie cardiaque}

Résumé : Le modèle bidomaine est le plus complet pour représenter la propagation des potentiels d'action cardiaque. Le modèle monodomaine est une simplification de celui-ci, moins coûteuse numériquement, mais valable seulement sous une hypothèse d'égale anisotropie. Nous proposons des approximations optimales de type monodomaine d'un modèle bidomaine fixé. Nous montrons d'abord que l'erreur entre les solutions du bidomaine et du monodomaine est bornée par la différence $\|B-A\|$ entre les opérateurs correspondants. Un opérateur monodomaine optimal doit donc minimiser la distance $\|B-A\|$, ce qui est équivalent dans $\mathbb{R}^{d}$ à minimiser l'erreur entre les symboles des opérateurs en norme $L^{p}$. On peut aussi comparer ces symboles dans une direction donnée, ce qui revient à comparer la propagation des ondes planes dans cette direction. Nous démontrons qu'un modèle monodomaine peut propager $d$ ondes planes d'un modèle bidomaine. Puis nous résolvons le problème de minimisation sur les symboles en norme $L^{\infty}$ et $L^{2}$. Dans le premier cas, on minimise donc l'erreur de propagation des ondes planes de manière uniforme sur les directions. Nous comparons ces approximations avec celles de la littérature sur deux séries de cas tests. D'abord avec des conditions aux limites périodiques pour retrouver les résultats démontrés dans $\mathbb{R}^{d}$, puis sur un domaine borné avec conditions de Neumann homogènes. Dans le premier cas, l'erreur de propagation est corrélée à la distance entre les symboles, alors que dans le second, les conditions aux limites dirigent le front et perturbent les résultats théoriques.

Mots-clés : Bidomaine, monodomaine, électrocardiologie 


\section{Introduction}

The bidomain model is currently accepted as the standard model for simulating the propagation of cardiac action potentials, $[16,23]$. Under the assumption of equal anisotropy ratio for the intra- and extra-cellular conductivities, this model degenerates into the simpler monodomain model,[9]. It is known that solving the monodomain model is about an order of magnitude cheaper in computational time than solving the bidomain model,[19, 24]. For this reason, several authors investigated the use of the monodomain model beyond the assumption of equal anisotropy ratio. Indeed comparisons of the bidomain and monodomain solutions were made, $[9,19,20]$. In these three studies, the conductivity tensor of the monodomain model was set equal to the harmonic average of the intra-and extra-cellular conductivities used in the bidomain model, and no external current source was used (avoiding the case of the virtual electrode). In each paper, solutions of both models were shown to be relatively close to each other. In Ref.[18], an optimization problem is proposed and solved while timestepping during computations to set the conductivity tensor in the equivalent monodomain model. Bidomain and monodomain solutions were shown to be closer to each other than usual monodomain approximations. However a theory has never been proposed for interpreting these positive results.

This paper proposes a theoretical framework for studying the error between bidomain and monodomain solutions. The difference between the bidomain and monodomain solutions will be related to the difference between the monodomain and bidomain linear operators as introduced in Ref. [5]. This difference is studied in the model problem $\Omega=\mathbb{R}^{d}$ with constant coefficients through Fourier transform and the symbol of the operators. The simplest interpretation of this study is in terms of travelling waves and how bidomain travelling waves are conserved by monodomain operators. The article is organized as follows. Section 2 presents the monodomain and bidomain models, and briefly the functional spaces and operators for properly setting these models. Section 3 states and proves the main comparison theorem between the monodomain and bidomain solutions. Section 4 introduces the concept of conserved planar waves between both models. Section 5 sets and analyzes an optimization problem that is derived from the comparison theorem and is used to define optimal monodomain approximations. In two subcases, this optimization problem is shown to have solutions. Connections are made between monodomain approximations and conserved planar waves. Finally, Section 6 illustrates with numerical test cases the relevance of the current theory.

\section{Mathematical models and functional setting}

\subsection{Bidomain and monodomain equations, comparison is- sues}

The bidomain equations are used to model the evolution of the transmembrane potential in an excitable tissue. They read

$$
\begin{aligned}
\partial_{t} u+f(u, v) & =-\operatorname{div}\left(\sigma^{(e)} \nabla u_{e}\right), \\
\partial_{t} v+g(u, v) & =0
\end{aligned}
$$


with

$$
-\operatorname{div}\left(\sigma^{(i)} \nabla u_{i}\right)-\operatorname{div}\left(\sigma^{(e)} \nabla u_{e}\right)=0,
$$

where $u_{i}$ and $u_{e}$ are the intra- and extra-cellular electric potential fields and $u=u_{i}-u_{e}$ is the transmembrane potential. In this model, the cardiomyocyte electrophysiology is described by nonlinear functions $f, g: \mathbb{R}^{2} \rightarrow \mathbb{R}$ and an additional unknown $v$, if one restricts to the simplest two-variable models such as the FitzHugh-Nagumo model, [13] or other models suited to myocardial cells, such as the Aliev-Panfilov,[1], the MacCulloch,[21] or the Mitchell-Schaeffer,[7] models. The electrical properties of the tissue are described by the intra- and extra-cellular conductivity tensors $\sigma^{(i)}$ and $\sigma^{(e)}$. The equations are usually written in a two- or three-dimensional bounded domain $\Omega \subset \mathbb{R}^{d}(d=2$ or 3 ) with boundary $\Gamma=\partial \Omega$ over which applies the following boundary conditions

$$
\sigma^{(i)} \nabla u_{i} \cdot \nu=0, \quad \sigma^{(e)} \nabla u_{e} \cdot \nu=0 \quad \text { on } \Gamma
$$

where $\nu$ denotes the unit outward normal to $\Gamma$. These homogeneous Neumann boundary conditions are commonly used for computing potential waves on hearts isolated from the thorax.

If the intra- and extra-cellular conductivities $\sigma^{(i)}$ and $\sigma^{(e)}$ are proportional, that is $\sigma^{(e)}(x)=k \sigma^{(i)}(x)$ for all $x \in \Omega$ and for a fixed $k \in \mathbb{R}$, then equation (3) simplifies to $-\operatorname{div}\left(\sigma^{(e)} \nabla u_{e}\right)=\frac{k}{k+1} \operatorname{div}\left(\sigma^{(i)} \nabla u\right)$. This results in the monodomain equations

$$
\begin{aligned}
& \partial_{t} u+f(u, v)=\operatorname{div}(\sigma \nabla u), \\
& \partial_{t} v+g(u, v)=0
\end{aligned}
$$

where the conductivity tensor is the harmonic average of the intra- and extracellular ones :

$$
\sigma=\frac{1}{k+1} \sigma^{(e)}=\frac{k}{k+1} \sigma^{(i)}=\left(\sigma^{(i)^{-1}}+\sigma^{(e)^{-1}}\right)^{-1}
$$

In this case, called "equal anisotropy ratio", the boundary conditions simplify to

$$
\sigma \nabla(u) \cdot \nu=0
$$

Although these equations are equivalent under the assumption $\sigma^{(i)}=k \sigma^{(e)}$ our aim is to provide comparisons between monodomain and bidomain solutions when this assumption does not hold. In this general setting, three questions will be answered:

- What is the error between the monodomain solutions with some conductivity $\sigma$ and the bidomain solutions with conductivities $\sigma^{(i)}$ and $\sigma^{(e)}$ ?

- What would be the optimal choice of $\sigma$ to minimize this error? Is it the harmonic average as in eq. (7)?

- How does planar travelling wave propagation compare between these bidomain and monodomain equations? 


\subsection{Bidomain and monodomain operators}

The monodomain model is a standard reaction-diffusion equation that can be analyzed for instance using the theory developped in Ref. [22], at least for the FitzHugh-Nagumo functions. The existence and uniqueness of solutions of the bidomain equations has been only recently studied by various authors, [25, 3, $4,5,10]$. We shall use the framework developped in Ref. [5] in which both equations are written in terms of uniformly elliptic operators that allows to enter the Hille-Yosida framework.

Excitable tissues are organized in fibers and fibers are organized in sheets[8]. It defines an orthonormal basis $\left(n_{1}, \ldots, n_{d}\right)$ at each point $x \in \bar{\Omega}$ where $n_{1}$ is the fiber's direction, $n_{2}$ is orthogonal to the fibers but tangent to the sheet while $n_{3}$ is orthogonal to the sheet (only in $3 \mathrm{D}$ ). The conductivity tensors are always assumed to be defined on $\bar{\Omega}$ and aligned with these directions. For the monodomain conductivity, it reads

$$
\forall x \in \bar{\Omega}, \quad \sigma(x)=\sum_{j=1}^{d} \sigma_{j}(x) n_{j}(x) n_{j}(x)^{T} .
$$

The intra- and extra-cellular conductivities are similarly characterized by their eigenvalues in the directions $\left(n_{j}\right)_{j=1, \ldots, d}$, specifically they are $\left(\sigma_{j}^{(i)}\right)_{j=1, \ldots, d}$ and $\left(\sigma_{j}^{(e)}\right)_{j=1, \ldots, d}$. In 3D, there is a special case called transversely isotropic in which the conductivities in the 2 directions perpendicular to the fiber are equal, $\sigma_{2}^{(i, e)}=$ $\sigma_{3}^{(i, e)}$, while the general case is called orthotropic.

The sheets of fibers are necessarily tangent to the boundary $\Gamma$ of $\Omega$ so that the normal $\nu$ is the last eigenvector of the conductivity tensors:

$$
\forall x \in \Gamma, \quad \sigma \nu=\sigma_{d} \nu, \quad \sigma^{(i)} \nu=\sigma_{d}^{(i)} \nu, \quad \sigma^{(e)} \nu=\sigma_{d}^{(e)} \nu
$$

The conductivity tensors are assumed to be bounded and uniformly elliptic: there exists $m>0$ and $M>0$ such that

$$
\forall x \in \bar{\Omega}, \quad m \leq \sigma_{j}(x) \leq M, \quad m \leq \sigma_{j}^{(i)}(x) \leq M, \quad m \leq \sigma_{j}^{(e)}(x) \leq M .
$$

Under these assumptions, the boundary conditions (4) and (8) both degenerate to the homogeneous Neumann conditions of the form $\nabla u \cdot \nu=0$ on $\Gamma$. Hence the monodomain operator $A u=-\operatorname{div}(\sigma \nabla u)$ and the intra- and extra-cellular operators $A_{i} u=-\operatorname{div}\left(\sigma^{(i)} \nabla u\right)$ and $A_{e} u=-\operatorname{div}\left(\sigma^{(e)} \nabla u\right)$ are well-defined,

- either as unbounded operators in $L^{2}(\Omega)$ with common domain $D=\left\{u \in H^{2}(\Omega), \nabla u \cdot \nu=0\right.$ a.e. in $\left.\Gamma\right\} \subset L^{2}(\Omega)$

- or as weak operators from $H^{1}(\Omega)$ onto its dual $\left(H^{1}(\Omega)\right)^{\prime}$.

They are maximal monotone and self-adjoint operators or similarly, uniformly elliptic second order differential operators. Denoting by $L^{2}(\Omega) / \mathbb{R}$ and $D / \mathbb{R}$ the spaces of functions defined up to a constant or equivalently functions with 0 average value on $\Omega$, the inverse operators $A_{i}^{-1}$ and $A_{e}^{-1}$ are well-defined on $L^{2}(\Omega) / \mathbb{R}$ with range $D / \mathbb{R}$. Consequently, the balance equation (3) can be solved for $u_{e}$ as a function of $u$ like it was done above to obtain the monodomain equations but this time without assuming proportional conductivities: $A_{i} u_{i}=-A_{e} u_{e}$ so 
that $A_{e} u_{e}=-A_{e} u+A_{e} u_{i}=A_{e} u-A_{e} A_{i}^{-1} A_{e} u_{e}$, thus $\left(A_{e}^{-1}+A_{i}^{-1}\right) A_{e} u_{e}=-u$. The bidomain equations can then be written as

$$
\begin{aligned}
\partial_{t} u+f(u, v)+B u & =0, \\
\partial_{t} v+g(u, v) & =0,
\end{aligned}
$$

where the bidomain operator $B$ is the harmonic average of the intra- and extracellular operators $A_{i}$ and $A_{e}$ instead of the operator induced by the harmonic average of the conductivities:

$$
B=\left(A_{i}^{-1}+A_{e}^{-1}\right)^{-1} .
$$

As explained in details in Ref. [5] this operator is well-defined on $D / \mathbb{R}$ and extended to $D$ into an operator that is still self-adjoint and uniformly elliptic, although it is not generally a differential operator.

Using similar notations, the monodomain equations rewrite as:

$$
\begin{aligned}
\partial_{t} u+f(u, v)+A u & =0, \\
\partial_{t} v+g(u, v) & =0 .
\end{aligned}
$$

For the bidomain equations, it is established in Ref. [5], existence and uniqueness of strong solutions (in $H^{2}(\Omega)$ ) local in time for any locally Lipschitz continuous nonlinearities $f$ and $g$, and existence of weak solutions (in $H^{1}(\Omega)$ ) global in time for nonlinearities used in electrocardiology, the FitzHugh-Nagumo,[13] the Aliev-Panfilov,[1] and the MacCulloch,[21] models. Uniqueness of global weak solution is shown only for the FitzHugh-Nagumo model.

\section{Comparison theorem between bidomain and monodomain solutions}

The introduction of the bidomain operator $B$ allows to compare naturally the solutions of the bidomain eqs. (11)-(12) and of the monodomain eqs (13)- (14).

thm 3.1 (Comparison in the energy norm). Given $\mu>0$, let $F_{\mu}: \mathbb{R}^{2} \rightarrow$ $\mathbb{R}^{2}$ denote the mapping defined by $F_{\mu}(z)=\left(\mu f\left(z_{1}, z_{2}\right), g\left(z_{1}, z_{2}\right)\right)$ for any $z=$ $\left(z_{1}, z_{2}\right) \in \mathbb{R}^{2}$. Suppose that there exist $\mu>0$ and $C \in \mathbb{R}$ such that

$$
\forall z \in \mathbb{R}^{2}, \quad \frac{1}{2}\left(\nabla F_{\mu}(z)+\nabla^{T} F_{\mu}(z)\right) \geq-C \operatorname{diag}(\mu, 1),
$$

where $A \geq B$ means that $x^{T} A x \geq x^{T} B x$ for all $x \in \mathbb{R}^{d}$. Assume that $\left(u_{m}, v_{m}\right)$ is a solution to the monodomain equations (13)- (14) and $\left(u_{b}, v_{b}\right)$ is a solution to the bidomain equations (11)-(12), both defined for $t \in[0, T]$ for some $T>0$ and with the same initial value,

$$
\forall x \in \Omega, \quad u_{b}(0, x)=u_{m}(0, x), \quad v_{b}(0, x)=v_{m}(0, x) .
$$

Strong solutions are defined in $C^{0}\left([0, T], H^{2}(\Omega)\right)$ but for $T>0$ small enough while weak solutions are defined in $C^{0}\left([0, T], L^{2}(\Omega)\right) \cap L^{2}\left(0, T ; H^{1}(\Omega)\right)$ but for all $T>0$. In any case, we have for all $t \in[0, T]$,

$$
\left(\mu\left\|u_{b}(t)-u_{m}(t)\right\|_{L^{2}(\Omega)}+\left\|v_{b}(t)-v_{m}(t)\right\|_{L^{2}(\Omega)}\right)^{1 / 2} \leq \gamma\|B-A\|_{\mathcal{L}} N_{T}\left(u_{b}\right) \mathrm{e}^{C T}
$$


with the notations

$$
\begin{gathered}
\gamma=\frac{1}{(2 C \mu)^{1 / 2}}, \quad \mathcal{L}=\mathcal{L}\left(D, L^{2}\right), \quad N_{T}\left(u_{b}\right)=\left(\int_{0}^{T}\left\|u_{b}(t)\right\|_{H^{2}(\Omega)}^{2} d t\right)^{1 / 2}, \\
\gamma=\frac{1}{(\mu m)^{1 / 2}}, \quad \mathcal{L}=\mathcal{L}\left(H^{1},\left(H^{1}\right)^{\prime}\right), \quad N_{T}\left(u_{b}\right)=\left(\int_{0}^{T}\left|u_{b}(t)\right|_{H^{1}(\Omega)}^{2} d t\right)^{1 / 2},
\end{gathered}
$$

respectively for strong and weak solutions.

Proof. Let $e=\left(u_{m}-u_{b}, v_{m}-v_{b}\right)=\left(e_{1}, e_{2}\right)$ denote the difference between the monodomain and bidomain solutions. A classical calculation shows that the function $e$ verifies for all $t \in[0, T]$,

$$
\begin{aligned}
\frac{1}{2} \frac{d}{d t}\left(\mu\left\|e_{1}\right\|_{L^{2}(\Omega)}^{2}+\right. & \left.\left\|e_{2}\right\|_{L^{2}(\Omega)}^{2}\right)+ \\
+ & \mu\left\langle A u_{m}-B u_{b}, u_{m}-u_{b}\right\rangle \\
& +\int_{\Omega} \mu\left(f\left(u_{m}, v_{m}\right)-f\left(u_{b}, v_{b}\right)\right)\left(u_{m}-u_{b}\right) \\
& +\int_{\Omega}\left(g\left(u_{m}, v_{m}\right)-g\left(u_{b}, v_{b}\right)\right)\left(v_{m}-v_{b}\right)=0
\end{aligned}
$$

where \langle\rangle stands either for the $\left(H^{1}\right)^{\prime} \times H^{1}$ duality product (weak solutions) or the $L^{2}$ dot product (strong solutions). The integrals with $f$ and $g$ make sense either because of the polynomial form of $f$ and $g$ or because these functions are locally Lipschitz as explained in Ref. [5]. Now, we write $A u_{m}-B u_{b}=A e_{1}-(B-A) u_{b}$ and the nonlinear part in terms of the function $F_{\mu}$ introduced above:

$$
\begin{aligned}
& \frac{1}{2} \frac{d}{d t}\left(\mu\left\|e_{1}\right\|_{L^{2}(\Omega)}^{2}+\left\|e_{2}\right\|_{L^{2}(\Omega)}^{2}\right)+\mu\left\langle A e_{1}, e_{1}\right\rangle+ \\
& \qquad \int_{\Omega}\left(F_{\mu}\left(u_{m}, v_{m}\right)-F_{\mu}\left(u_{b}, v_{b}\right)\right) \cdot e=\left\langle(B-A) u_{b}, e_{1}\right\rangle .
\end{aligned}
$$

Remark that,

$$
\left(F_{\mu}\left(u_{m}, v_{m}\right)-F_{\mu}\left(u_{b}, v_{b}\right)\right)=\int_{0}^{1} \nabla F_{\mu}(z(\theta)) e d \theta
$$

for $z(\theta)=\theta\left(u_{m}, v_{m}\right)+(1-\theta)\left(u_{b}, v_{b}\right)$ and then

$$
\int_{\Omega}\left(F_{\mu}\left(u_{m}, v_{m}\right)-F_{\mu}\left(u_{b}, v_{b}\right)\right) \cdot e \geq-C\left(\mu\left\|e_{1}\right\|_{L^{2}(\Omega)}^{2}+\left\|e_{2}\right\|_{L^{2}(\Omega)}^{2}\right) .
$$

As a consequence, using the ellipticity condition (10),

$$
\begin{aligned}
& \frac{1}{2} \frac{d}{d t}\left(\mu\left\|e_{1}\right\|_{L^{2}(\Omega)}^{2}+\left\|e_{2}\right\|_{L^{2}(\Omega)}^{2}\right)+\mu m\left|e_{1}\right|_{H^{1}(\Omega)}^{2} \\
\leq & C\left(\mu\left\|e_{1}\right\|_{L^{2}(\Omega)}^{2}+\left\|e_{2}\right\|_{L^{2}(\Omega)}^{2}\right)+ \begin{cases}D\left\|u_{b}\right\|_{H^{2}(\Omega)}\left\|e_{1}\right\|_{L^{2}(\Omega)} & \text { for strong solutions } \\
D\left|u_{b}\right|_{H^{1}(\Omega)}\left|e_{1}\right|_{H^{1}(\Omega)} & \text { for weak solutions }\end{cases}
\end{aligned}
$$

where $D=\|B-A\|_{\mathcal{L}\left(D, L^{2}\right)}$ for strong solutions and $D=\|B-A\|_{\mathcal{L}\left(H^{1},\left(H^{1}\right)^{\prime}\right)}$ for weak solutions. Defining $D(t)=D\left\|u_{b}(t)\right\|_{H^{2}(\Omega)}$ for strong solutions, $D(t)=$ 
$D\left|u_{b}(t)\right|_{H^{1}(\Omega)}$ for weak solutions and $Y(t)=\left(\mu\left\|e_{1}\right\|_{L^{2}(\Omega)}^{2}+\left\|e_{2}\right\|_{L^{2}(\Omega)}^{2}\right)^{1 / 2}$, we have, for all $t \in[0, T]$,

$$
\frac{d}{d t} Y(t)^{2} \leq 2 C Y(t)^{2}+2 \frac{D(t)}{\mu^{1 / 2}} Y(t)
$$

for strong solutions, because $\mu\left\|e_{1}\right\|_{L^{2}}^{2} \leq Y(t)^{2}$ and

$$
\frac{d}{d t} Y(t)^{2} \leq 2 C Y(t)^{2}+\frac{D(t)^{2}}{\mu m}
$$

for weak solutions because $2 D(t)\left|e_{1}\right|_{H^{1}} \leq \mu m\left|e_{1}\right|_{H^{1}(\Omega)}^{2}+\frac{D(t)^{2}}{\mu m}$. For strong solutions, a common argument to obtain Gronwall inequalities (see for instance the proof of lemma 3.11, Ref.[11], page 69) proves that

$$
\begin{aligned}
\forall t \in[0, T], \quad Y(t) \leq Y(0) \mathrm{e}^{C t}+\frac{1}{\mu^{1 / 2}} \int_{0}^{t} D(s) \mathrm{e}^{C(t-s)} d s \\
\leq \frac{1}{\mu^{1 / 2}}\|B-A\|_{\mathcal{L}}\left\|u_{b}\right\|_{L^{2}\left(0, t ; H^{2}(\Omega)\right)}\left(\frac{\mathrm{e}^{2 C t}-1}{2 C}\right)^{1 / 2}
\end{aligned}
$$

(using the Cauchy-Schwarz inequality and $Y(0)=0$ ). For weak solutions, a similar computation yields

$$
\begin{aligned}
\forall t \in[0, T], \quad Y(t)^{2} \leq Y(0)^{2} \mathrm{e}^{2 C t} & +\frac{1}{\mu m} \int_{0}^{t} D(s)^{2} \mathrm{e}^{2 C(t-s)} d s \\
& \leq \frac{1}{\mu m}\|B-A\|_{\mathcal{L}}^{2} \int_{0}^{t}\left|u_{b}(s)\right|_{H^{1}(\Omega)}^{2} d s \mathrm{e}^{2 C t} .
\end{aligned}
$$

As a conclusion, denoting $N_{T}\left(u_{b}\right)=\left\|u_{b}\right\|_{L^{2}\left(0, T ; H^{2}(\Omega)\right)}$ for strong solutions and $N_{T}\left(u_{b}\right)=\left(\int_{0}^{T}\left|u_{b}(s)\right|_{H^{1}(\Omega)}^{2} d s\right)^{1 / 2}$ for weak solutions, we always have

$$
Y(t) \leq \gamma\|B-A\|_{\mathcal{L}} N_{T}\left(u_{b}\right) \mathrm{e}^{C T} \quad \text { with } \gamma= \begin{cases}\frac{1}{(2 C \mu)^{1 / 2}} & \text { for strong solutions } \\ \frac{1}{(\mu m)^{1 / 2}} & \text { for weak solutions. }\end{cases}
$$

rmk 3.1. The monotonicity condition (15) is a natural hypothesis to prove the uniqueness of the solution for the bidomain model. It is proved for the FitzHughNagumo model,[5] for some $C>0$.

\section{Conservation of planar waves}

In this section, travelling waves are considered. We assume that $\Omega=\mathbb{R}^{d}$ and that $\left(u_{m}, v_{m}\right)$ and $\left(u_{b}, v_{b}\right)$ are classical solutions of the monodomain eqs. (13)-(14) and bidomain eqs. (11)-(12), respectively. Specifically, $u_{m}$ and $u_{b}$ are $C^{2}\left(\mathbb{R}^{d}\right)$ functions of $x$ for all $t$ and $C^{1}\left(\mathbb{R}^{+}\right)$functions of $t$ for all $x \in \mathbb{R}^{d}$; while $v_{m}$ and $v_{b}$ are $C^{0}\left(\mathbb{R}^{d}\right)$ functions of $x$ and $C^{1}\left(\mathbb{R}^{+}\right)$functions of $t$. 
def 4.1 (Planar waves). Given a direction vector $n \in \mathbb{S}^{d-1}:=\left\{n \in \mathbb{R}^{d},|n|=\right.$ $1\}$, planar waves are solutions that depends only on one space dimension, $x$. $n$. They include in particular travelling waves of the form $u(x, t)=\bar{u}(x \cdot n-$ $c t)$. More generally here, a planar wave solution $\left(u_{m}, v_{m}\right)$ for the monodomain equations (13)-(14) is of the form

$$
\forall(x, t) \in \mathbb{R}^{d} \times \mathbb{R}^{+}, \quad u_{m}(x, t)=\bar{u}(x \cdot n, t), \quad v_{m}(x, t)=\bar{v}(x \cdot n, t),
$$

for some functions $\bar{u}$ of class $C^{1}$ in $t$ and $C^{2}$ in $x$ and $\bar{v}$ of class $C^{1}$ in $t$ and $C^{0}$ in $x$. Planar waves $\left(u_{b}, v_{b}\right)$ for the bidomain eqs. (11)-(12) are defined similarly. These sets of planar waves solutions are denoted by $P W_{m}(n)$ and $P W_{b}(n)$, respectively, for the monodomain and the bidomain equations.

For the bidomain equations, it is sufficient to define $u_{b}$ and $v_{b}$ since the equation (3) together with $u_{b}=u_{i}-u_{e}$ exactly defines the functions $u_{i}$ and $u_{e}$. Specifically, if $\left(u_{b}, v_{b}\right) \in \mathrm{PW}_{b}(n)$ then $u_{e}(x, t)=\bar{u}_{e}(x \cdot n, t)$ is solution of

$$
-\operatorname{div}\left(\left(\sigma^{(i)}+\sigma^{(e)}\right) \nabla u_{e}\right)=\operatorname{div}\left(\sigma^{(i)} \nabla u\right)
$$

that reduces to a linear ordinary differential equation in $x \cdot n$ for $\bar{u}_{e}$.

def 4.2 (Conservation of planar waves). The monodomain equations associated to $\sigma$ are said to conserve the planar waves of the bidomain equations associated to $\sigma^{(i)}$ and $\sigma^{(e)}$ in the direction $n \in \mathbb{S}^{d-1}$ iff both systems of equations have the same planar wave solutions in the direction $n$, namely $P W_{m}(n)=P W_{b}(n)$.

thm 4.1. Assume that $\Omega=\mathbb{R}^{d}$, and that the matrices $\sigma^{(i)}, \sigma^{(e)}$ and $\sigma$ are independent of $x \in \mathbb{R}^{d}$ and have their eigenvectors aligned with the main axes of $\mathbb{R}^{d}$ :

$$
\sigma^{(i)}=\operatorname{diag}\left(\sigma_{1}^{(i)}, \ldots, \sigma_{d}^{(i)}\right), \quad \sigma^{(e)}=\operatorname{diag}\left(\sigma_{1}^{(e)}, \ldots, \sigma_{d}^{(e)}\right), \quad \sigma=\operatorname{diag}\left(\sigma_{1}, \ldots, \sigma_{d}\right)
$$

with $\sigma_{j}^{(i, e)}>0$ and $\sigma_{j}>0, j=1 \ldots, d$. The monodomain equations with conductivity $\sigma$ conserve the planar waves of the bidomain equations with conductivities $\sigma^{(i)}$ and $\sigma^{(e)}$ in the direction $n$ iff

$$
s(n)=\left(s_{i}(n)^{-1}+s_{e}(n)^{-1}\right)^{-1}
$$

where

$$
s_{i}(n)=n^{T} \sigma^{(i)} n, \quad s_{e}(n)=n^{T} \sigma^{(e)} n, \quad s(n)=n^{T} \sigma n .
$$

Proof. Indeed for any $n \in \mathbb{S}^{d-1}$ and any regular function of the form $u(x, t)=$ $\bar{u}(x \cdot n, t)$, we have

$$
\operatorname{div}(\sigma \nabla u(x, t))=s(n) \partial_{\xi \xi} \bar{u}(\xi, t), \quad \operatorname{div}\left(\sigma^{(i, e)} \nabla u(x, t)\right)=s_{i, e}(n) \partial_{\xi \xi} \bar{u}(\xi, t),
$$

where $\partial_{\xi \xi} \bar{u}$ denotes the second order partial derivative of $\bar{u}$ along $\xi=x \cdot n$. The coefficients $s_{i}, s_{e}$ and $s$ are constant, thus the set $\mathrm{PW}_{m}(n)$ is defined by regular solutions of the equations

$$
\begin{gathered}
\partial_{t} \bar{u}(\xi, t)+f(\bar{u}(\xi, t), \bar{v}(\xi, t))=s(n) \partial_{\xi \xi} \bar{u}(\xi, t), \\
\partial_{t} \bar{v}(\xi, t)+g(\bar{u}(\xi, t), \bar{v}(\xi, t))=0
\end{gathered}
$$


while the set $\mathrm{PW}_{b}(n)$ is defined by regular solutions to the equations

$$
\begin{gathered}
\partial_{t} \bar{u}(\xi, t)+f(\bar{u}(\xi, t), \bar{v}(\xi, t))=-s_{e}(n) \partial_{\xi \xi} \bar{u}_{e}(\xi, t), \\
-\left(s_{i}(n)+s_{e}(n)\right) \partial_{\xi \xi} \bar{u}_{e}(\xi, t)=s_{i}(n) \partial_{\xi \xi} \bar{u}(\xi, t), \\
\partial_{t} \bar{v}(\xi, t)+g(\bar{u}(\xi, t), \bar{v}(\xi, t))=0,
\end{gathered}
$$

which is equivalent with

$$
\begin{gathered}
\partial_{t} \bar{u}(\xi, t)+f(\bar{u}(\xi, t), \bar{v}(\xi, t))=\left(s_{i}(n)^{-1}+s_{e}(n)^{-1}\right)^{-1} \partial_{\xi \xi} \bar{u}(\xi, t), \\
-\left(s_{i}(n)+s_{e}(n)\right) \partial_{\xi \xi} \bar{u}_{e}(\xi, t)=s_{i}(n) \partial_{\xi \xi} \bar{u}(\xi, t), \\
\partial_{t} \bar{v}(\xi, t)+g(\bar{u}(\xi, t), \bar{v}(\xi, t))=0 .
\end{gathered}
$$

This concludes the proof.

\section{Optimization problems and approximate monodomain operators}

Given a bidomain system of equations associated to conductivities $\sigma^{(i)}$ and $\sigma^{(e)}$, the problem is now to find a conductivity $\sigma$ that either minimizes the operator norm $\|B-A\|_{\mathcal{L}}$ or conserves the planar waves solutions in as many directions as possible. Both problems have solutions and are quite similar.

We use the theory of pseudo-differential operators, [2] to identify the optimization problem. Again, assume that $\Omega=\mathbb{R}^{d}$ and the conduction tensors $\sigma^{(i)}$, $\sigma^{(e)}$ and $\sigma$ have constant coefficients and are aligned with the main axes of $\mathbb{R}^{d}$, so that they read as in eq. (17). Their eigenvalues are denoted by $\sigma=\left(\sigma_{1}, \ldots, \sigma_{d}\right)$ and $\sigma \geq 0$ (resp. $\sigma>0$ ) means $\sigma_{j} \geq 0$ (resp. $\sigma_{j}>0$ ) for all $j=1, \ldots, d$. The symbols of the operators $A_{i}, A_{e}$ and $A$ defined in section 2.2 are the functions in $\mathbb{R}^{d}$ defined by

$$
S_{i}(\xi)=\sum_{j=1}^{d} \sigma_{j}^{(i)}\left|\xi_{j}\right|^{2}, \quad S_{e}(\xi)=\sum_{j=1}^{d} \sigma_{j}^{(e)}\left|\xi_{j}\right|^{2}, \quad S_{\sigma}(\xi)=\sum_{j=1}^{d} \sigma_{j}\left|\xi_{j}\right|^{2},
$$

for $\xi=\left(\xi_{1}, \ldots, \xi_{d}\right) \in \mathbb{R}^{d}$. Using the fact that the tensors $\sigma^{(i)}$ and $\sigma^{(e)}$ are constant over $\Omega$, the symbol of the bidomain operator $B$ is

$$
H(\xi)=\left(S_{i}^{-1}(\xi)+S_{e}^{-1}(\xi)\right)^{-1}=\frac{S_{i}(\xi) S_{e}(\xi)}{S_{i}(\xi)+S_{e}(\xi)} .
$$

The restrictions to $\mathbb{S}^{d-1}$ of these symbols are exactly the 1D conductivity coefficients $s_{i}(n), s_{e}(n)$ and $s(n)$ that appear while planar waves of the bidomain and monodomain equations are conserved in thm 4.1. As a consequence, the monodomain equations associated to $\sigma \in \mathbb{R}^{d}$ conserve the planar waves of the bidomain equations associated to $\sigma^{(i)}$ and $\sigma^{(e)}$ in the direction $n \in \mathbb{S}^{d-1}$ iff $H(n)=S_{\sigma}(n)$.

All the symbols considered depend only on the $\left(\xi_{j}\right)^{2}$ 's so that the problem can be reformulated with the change of variable $x_{j}=\left(\xi_{j}\right)^{2}$ for $j=1, \ldots, d$. Then the set $\mathbb{S}^{d-1}$ is mapped to the simplex

$$
\Sigma=\left\{x=\left(x_{1}, \ldots, x_{d}\right) \in \mathbb{R}^{d}, \quad x_{j} \geq 0, \quad \sum_{j=1}^{d} x_{j}=1\right\} \subset \mathbb{R}^{d} .
$$

$\mathrm{RR} \mathrm{n}^{\circ} 7810$ 
In the following, we note by $a_{j}=\left(\delta_{j k}\right)_{k=1, \ldots, d}$ the vertices of $\Sigma$. The symbols are replaced by the functions

$$
\begin{gathered}
S_{i}(x)=\sigma^{(i)} \cdot x, \quad S_{e}(x)=\sigma^{(e)} \cdot x, \\
H(x)=\frac{\left(\sigma^{(i)} \cdot x\right)\left(\sigma^{(e)} \cdot x\right)}{\left(\sigma^{(i)}+\sigma^{(e)}\right) \cdot x}, \quad S_{\sigma}(x)=\sigma \cdot x .
\end{gathered}
$$

In particular, the symbols $S_{i}, S_{e}$ and $S_{\sigma}$ extend to linear operators in $x \in \mathbb{R}^{d}$.

rmk 5.1. The parameterization $x$ is simpler to carry out the proofs below, but it is necessary to keep in mind the original parameterization $\xi \in \mathbb{S}^{d-1}$ because it has a practical meaning: comparing $H(\xi)$ and $S_{\sigma}(\xi)$ amounts to compare the planar waves that propagates in the physical direction $\xi$. Note that a direction $x=\left(x_{1}, \ldots, x_{d}\right) \in \Sigma$ is associated to at most $2^{d}$ directions $\xi$ on the unit sphere $\mathbb{S}^{d-1}$, specifically $\left( \pm \sqrt{x_{1}}, \ldots, \pm \sqrt{x_{d}}\right)$.

If the function $H$ is linear with positive coefficients in $\Sigma$, meaning that $H(x)=\sigma \cdot x$ with $\sigma=\left(\sigma_{1}, \ldots, \sigma_{d}\right)>0$, then it is the symbol of the monodomain operator $S_{\sigma}$. The theorem below shows that the equal anisotropy ratio assumption is a necessary and sufficient condition for $H$ to be linear.

thm 5.1. The function $H(x)$ is concave on $\Sigma$. Furthermore, $H(x)$ is linear on $\Sigma$ iff there exists $k \in \mathbb{R}$ such that $\sigma^{(i)}=k \sigma^{(e)}$. In this case, $H(x)$ is the symbol of the usual harmonic monodomain approximation: $H(x)=\sigma \cdot x$ with $\sigma=\left(\sigma_{1}, \ldots, \sigma_{d}\right)$ and $\sigma_{j}=\left(\sigma_{j}^{(i)^{-1}}+\sigma_{j}^{(e)^{-1}}\right)^{-1}>0$ for all $j=1, \ldots, d$.

Proof. Remark that $H=F \circ G$ where $F(s, t)=\frac{s t}{s+t}$ and $G(x)=\left(\sigma^{(i)} \cdot x, \sigma^{(e)} \cdot x\right)$ is a linear function such that $G(x) \geq 0$ on $\Sigma$. The Hessian matrix of $F$ is

$$
\nabla^{2} F(s, t)=\frac{2}{(s+t)^{3}}\left(\begin{array}{cc}
-t^{2} & s t \\
s t & -s^{2}
\end{array}\right)=-\frac{2}{(s+t)^{3}}\left(\begin{array}{c}
-t \\
s
\end{array}\right)\left(\begin{array}{ll}
-t & s
\end{array}\right) .
$$

This matrix is negative semi-definite for $s \geq 0$ and $t \geq 0$. Hence, the function $F$ is concave so that its composition with a linear function is still concave. A straightforward computation shows that

$$
\nabla^{2} H(x)=\left(\begin{array}{ll}
\sigma^{(i)} & \sigma^{(e)}
\end{array}\right) \nabla^{2} F\left(S_{i}(x), S_{e}(x)\right)\left(\begin{array}{l}
\sigma^{(i)^{T}} \\
\sigma^{(e)^{T}}
\end{array}\right)
$$

and finally,

$$
\begin{gathered}
\nabla^{2} H(x)=-\frac{2}{\left(S_{i}(x)+S_{e}(x)\right)^{3}}\left(S_{e}(x) \sigma^{(i)}-S_{i}(x) \sigma^{(e)}\right)\left(S_{e}(x) \sigma^{(i)^{T}}-S_{i}(x) \sigma^{(e)^{T}}\right) \\
=-\frac{2}{\left(S_{i}(x)+S_{e}(x)\right)^{3}}\left(\sigma^{(i)} \sigma^{(e)^{T}}-\sigma^{(e)} \sigma^{(i)^{T}}\right) x x^{T}\left(\sigma^{(e)} \sigma^{(i)^{T}}-\sigma^{(i)} \sigma^{(e)^{T}}\right)
\end{gathered}
$$

because $S_{i}(x)=\sigma^{(i)^{T}} x=x^{T} \sigma^{(i)}$ and $S_{e}(x)=\sigma^{(e)^{T}} x=x^{T} \sigma^{(e)}$. Let $A=$ $\sigma^{(e)} \sigma^{(i)^{T}}-\sigma^{(i)} \sigma^{(e)^{T}}$. Then

$$
\forall x \in \Sigma, \nabla^{2} H(x)=0 \Leftrightarrow \forall x \in \Sigma, x^{T} A=0 \Leftrightarrow A=0
$$


because $\operatorname{span}(\Sigma)=\mathbb{R}^{d}$. Consequently,

$$
\forall x \in \Sigma, \nabla^{2} H(x)=0 \Leftrightarrow \sigma^{(i)} \sigma^{(e)^{T}}=\sigma^{(e)} \sigma^{(i)^{T}} \Leftrightarrow \exists k \in \mathbb{R}, \sigma^{(i)}=k \sigma^{(e)},
$$

where the last equivalence follows from the fact that $\sigma^{(i)}$ and $\sigma^{(e)}$ are diagonalizable in the same basis. Thus, the function $H$ is linear on $\Sigma$ iff $\sigma^{(i)}=k \sigma^{(e)}$ for some $k \in \mathbb{R}$. In this case,

$$
H(x)=\frac{\left(\sigma^{(i)} \cdot x\right)\left(\sigma^{(e)} \cdot x\right)}{\left(\sigma^{(i)}+\sigma^{(e)}\right) \cdot x}=\frac{k}{1+k} \sigma^{(e)} \cdot x=\sigma \cdot x
$$

which implies that $\sigma$ is as given in the statement of the theorem.

Now we wonder if there exists a monodomain operator that conserves the planar waves in $d$ given independent directions. It amounts to built the linear function that interpolates the function $H$ in $d$ independent directions.

From the previous theorem, this question is already answered if the directions in $\mathbb{S}^{d-1}$ correspond to the vertices $a_{j}$ of $\Sigma$. In this case, the conductivities $\sigma_{j}$ in the monodomain operator are the harmonic averages of the bidomain conductivity coefficients. Consequently, the so-called harmonic monodomain conserves the planar waves in the principal directions of the intra- and extracellular conductivity tensors, namely the fiber and cross-fiber directions $n_{j}$, $j=1, \ldots, d$, defined in sec. 2.2 .

For general directions, the answer is more subtle: the coefficients of the linear interpolate might not define a monodomain operator in the sense that $\sigma$ might be indefinite.

thm 5.2. Given d linearly independent directions $x_{1}, \ldots, x_{d}$ in $\Sigma$, there exists a unique $\sigma \in \mathbb{R}^{d}$ such that $S_{\sigma}\left(x_{j}\right)=H\left(x_{j}\right)$ for all $j=1, \ldots, d$, where $S_{\sigma}(x)=\sigma \cdot x$. For the positivity of $\sigma$, the following holds:

i) If $d=2$, then $\sigma>0$ for any choice of independent points $x_{1}, x_{2}$, so that it defines a monodomain operator equivalent to the bidomain one in the 2 directions $x_{1}$ and $x_{2}$.

ii) If $d=3$, then it is not possible to guarantee $\sigma>0$, even $\sigma$ indefinite is possible.

Proof. Given $x_{1}, \ldots, x_{d} \in \Sigma$, let $\left(x_{j k}\right)_{k=1 \ldots d}$ denote the coordinates of $x_{j} \in \Sigma$. The problem $S_{\sigma}\left(x_{j}\right)=H\left(x_{j}\right)$ for $j=1, \ldots, d$ is a linear system of $d$ equations with $d$ unknowns, specifically, $\sum_{k=1}^{d} x_{j k} \sigma_{k}=H\left(x_{j}\right)$ for $j=1, \ldots, d$. This problem has a unique solution $\sigma \in \mathbb{R}^{d}$ because the directions $x_{j}$ are linearly independent.

If $d=2$, it is clear that the graph of the solution $S_{\sigma}(x)$ is the secant of $H$ through the points $\left(x_{1}, H\left(x_{1}\right)\right)$ and $\left(x_{2}, H\left(x_{2}\right)\right)$. Thus, either $H$ is affine and then $\sigma_{j}=\left(\sigma_{j}^{(i)^{-1}}+\sigma_{j}^{(e)^{-1}}\right)^{-1}$ or it is concave and $\sigma_{j} \geq\left(\sigma_{j}^{(i)}{ }^{-1}+\sigma_{j}^{(e)^{-1}}\right)^{-1}=$ $H\left(a_{j}\right)$, where $a_{j}$ denotes any vertex of $\Sigma$.

If $d=3$, the positivity of $\sigma$ is not guaranteed. For instance, for $\sigma^{(i)}=$ $(1,5,1), \sigma^{(e)}=(1,1,5), x_{1}=(1 / 2,1 / 2,0), x_{2}=(1 / 2,0,1 / 2)$ and $x_{3}=(1 / 3,1 / 3$, $1 / 3)$, a short computation shows that $\sigma=(-1 / 2,2,2)$ is the only solution of $S_{\sigma}\left(x_{j}\right)=H\left(x_{j}\right)$ for $j=1,2,3$, but with $\sigma_{1}<0$. 
This theorem is an interpolation result, while the comparison between the monodomain and bidomain operators will lead to an approximation problem for the same functions. Specifically, for any $u \in D$, the Fourier transform of $B u-A u$ is given by

$$
\mathcal{F}(B u)(\xi)-\mathcal{F}(A u)(\xi)=\left(H(\xi)-S_{\sigma}(\xi)\right) \mathcal{F}(u)(\xi),
$$

The norm $\|A-B\|_{\mathcal{L}}$ is the norm of $A-B$ as an operator from $H^{k}$ to $H^{k-2}$. It is computed through Fourier transform:

$$
\begin{aligned}
&\|B u-A u\|_{H^{k-2}\left(\mathbb{R}^{d}\right)}^{2}=\int_{\mathbb{R}^{d}}\left|H(\xi)-S_{\sigma}(\xi)\right|^{2}\left(1+|\xi|^{2}\right)^{k-2}|\mathcal{F}(u)(\xi)|^{2} d \xi \\
& \leq \sup _{\xi \in \mathbb{R}^{d}} \frac{\left|H(\xi)-S_{\sigma}(\xi)\right|^{2}}{\left(1+|\xi|^{2}\right)^{2}} \int_{\mathbb{R}^{d}}\left(1+|\xi|^{2}\right)^{k}|\mathcal{F}(u)(\xi)|^{2} d \xi \\
& \leq \sup _{\xi \in \mathbb{R}^{d}} \frac{\left|H(\xi)-S_{\sigma}(\xi)\right|^{2}}{|\xi|^{4}} \int_{\mathbb{R}^{d}}\left(1+|\xi|^{2}\right)^{k}|\mathcal{F}(u)(\xi)|^{2} d \xi \\
& \leq \sup _{|\xi|=1}\left|H(\xi)-S_{\sigma}(\xi)\right|^{2}|u|_{H^{k}\left(\mathbb{R}^{d}\right)}^{2},
\end{aligned}
$$

because $H$ and $S$ are homogeneous functions of degree 2. Hence, for both weak and strong solutions,

$$
\|B-A\|_{\mathcal{L}} \leq \sup _{|\xi|=1}\left|H(\xi)-S_{\sigma}(\xi)\right|=\max _{x \in \Sigma}\left|H(x)-S_{\sigma}(x)\right| .
$$

rmk 5.2. Replacing the term $\left(1+|\xi|^{2}\right)^{2}$ by $|\xi|^{4}$ at the denominator for bounding the error on the symbols is valid for all $\xi$ but the upper bound gets sharper as $|\xi| \rightarrow+\infty$, i.e. when the wave length becomes shorter.

rmk 5.3. The comparison theorem bounding the error between the bidomain and monodomain solutions by the error $\|A-B\|_{\mathcal{L}}$ is stated for solutions in $H^{k}(\Omega)$, for $k=1,2$, with $\Omega$ a bounded domain. These restrictions on $k$ and $\Omega$ come from the existence results in Ref. [5]. The argument above for bounding $\|A-B\|_{\mathcal{L}}$ by the error on the symbols is done for $\Omega=\mathbb{R}^{d}$ and must work for planar waves. Strictly speaking, the validity of this bound requires extending the existence result to solutions defined on the whole space $\mathbb{R}^{d}$ that are less regular, for instance with $k<0$.

The monodomain associated to $\sigma>0$ is optimal if its symbol minimizes its $L^{\infty}$ distance to the symbol of the bidomain operator. Our generalized optimality condition requires that it minimizes the $L^{p}$ distance

$$
J(\sigma)=\left\|H-S_{\sigma}\right\|_{L^{p}\left(\mathbb{S}^{d-1}\right)} .
$$

for some $1 \leq p \leq+\infty$. This minimization problem has a solution over $\{\sigma \geq$ 0\} $\subset \mathbb{R}^{d}$ which is a closed and convex subset of $\mathbb{R}^{d}$. The solution is unique for $1<p<\infty$. It remains to show that the minimizer verifies $\sigma>0$ for the associated operator to be a uniformly elliptic second order partial differential operator.

Solving this optimization problem amounts to identify a monodomain operator whose action on planar waves propagating in directions $\xi$ matches most closely the action of the bidomain operator in the sense of the $L^{p}$ norm. 


\subsection{The case $p=\infty$, approximation uniform over all di- rections}

Our problem in $L^{\infty}$ norm is to find $\sigma=\left(\sigma_{1}, \ldots, \sigma_{d}\right) \in \mathbb{R}^{d}$ such that $J(\sigma)=$ $\max _{x \in \Sigma}|H(x)-\sigma \cdot x|$ is minimum over $\mathbb{R}^{d}$.

Let $h_{j}=H\left(a_{j}\right)$ be the values of $H$ at the vertices of $\Sigma$ and $h=\left(h_{1}, \ldots, h_{d}\right) \in$ $\mathbb{R}^{d}$. Our problem is equivalent to look for $\sigma$ that minimizes

$$
J(\sigma)=\max _{x \in \Sigma}|H(x)-h \cdot x-\sigma \cdot x|=\max _{x \in \sigma}\left|H_{0}(x)-\sigma \cdot x\right|
$$

where the shifted function $H_{0}(x)=H(x)-h \cdot x$ is concave on $\Sigma$ and such that $H_{0}\left(a_{j}\right)=0$ for $j=1, \ldots, d$.

thm 5.3. Consider a function $H_{0}$ defined on $\Sigma$, continuous, concave and such that $H_{0}\left(a_{j}\right)=0$ for $j=1, \ldots, d$. We denote by $x^{\star} \in \Sigma$ a point where $H_{0}$ is maximum:

$$
H_{0}\left(x^{\star}\right)=\max _{x \in \Sigma} H_{0}(x):=\delta .
$$

Then $\sigma_{\delta / 2}=\left(\frac{\delta}{2}, \ldots, \frac{\delta}{2}\right)$ minimizes $J(\sigma)=\max _{x \in \Sigma}\left|H_{0}(x)-\sigma \cdot x\right|$ over $\mathbb{R}^{d}$. Furthermore, if there exists at least one minimizer $x^{\star}$ of $H_{0}$ with $x_{j}^{\star}>0$ for all $j=1, \ldots, d$ (that is $\left.x^{\star} \notin \partial \Sigma\right)$, then $\sigma_{\delta / 2}=\left(\frac{\delta}{2}, \ldots, \frac{\delta}{2}\right)$ is the unique minimizer of $J$.

Proof. Note that $\sigma_{\delta / 2} \cdot x=\frac{\delta}{2}$ for all $x \in \Sigma$. Since $H_{0}\left(a_{j}\right)=0$ for $j=1, \ldots, d$, since $H_{0}$ is concave and reaches its maximum at $x^{\star} \in \Sigma$, we have $0 \leq H_{0}(x) \leq \delta$ and then

$$
\forall x \in \Sigma, \quad-\frac{\delta}{2} \leq H_{0}(x)-\frac{\delta}{2} \leq \frac{\delta}{2} .
$$

We note that $H_{0}(x)-\frac{\delta}{2}=-\frac{\delta}{2}$ for $x=a_{j}$ and $H_{0}(x)-\frac{\delta}{2}=\frac{\delta}{2}$ for $x=x^{\star}$. Consequently, $J\left(\sigma_{\delta / 2}\right)=\frac{\delta}{2}$. Now suppose that $\frac{\delta}{2}$ is not the minimum of $J$. Then there exists $\sigma \in \mathbb{R}^{d}$ such that $J\left(\sigma_{\delta / 2}+\sigma\right)<\frac{\delta}{2}$. For $x=a_{j}(j=1, \ldots, d)$, we have $H_{0}(x)-\frac{\delta}{2}-\sigma \cdot x=-\frac{\delta}{2}-\sigma_{j}$ so that necessarily, $-\frac{\delta}{2}<-J\left(\sigma_{\delta / 2}+\sigma\right) \leq$ $-\frac{\delta}{2}-\sigma_{j} \leq J\left(\sigma_{\delta / 2}+\sigma\right)<\frac{\delta}{2}$ which means that

$$
-\delta<\sigma_{j}<0, \quad j=1, \ldots, d .
$$

This implies in particular that $\sigma \cdot x=\sum_{j=1}^{d} \sigma_{j} x_{j}<0$ for all $x \in \Sigma$. But for $x=x^{\star}$, we have $H_{0}(x)-\frac{\delta}{2}-\sigma \cdot x=\frac{\delta}{2}-\sigma \cdot x^{\star}$ and necessarily, $-\frac{\delta}{2}<\frac{\delta}{2}-\sigma \cdot x^{\star}<\frac{\delta}{2}$ which shows that $\sigma \cdot x^{\star}>0$. This is a contradiction so that $\frac{\delta}{2}$ is the minimum of $J$ and $\sigma_{\delta / 2}$ is a minimizer of $J$.

Now, suppose that $\sigma=\left(\sigma_{1}, \ldots, \sigma_{d}\right) \in \mathbb{R}^{d}$ is such that $\sigma_{\delta / 2}+\sigma$ is another minimizer for $J$. Then, using arguments similar to those above,

$$
-\delta \leq \sigma_{j} \leq 0, \quad \text { and } \sigma \cdot x^{\star}=0 .
$$

Consequently, if $x_{j}^{\star}>0$ for all $j=1, \ldots, d$ then $\sigma_{j}=0$ for all $j=1, \ldots, d$ and then $\sigma_{\delta / 2}$ is the unique minimizer of $J$ in $\mathbb{R}^{d}$.

rmk 5.4. If all $x_{j}^{\star}$ that maximize $H$ are on the boundary of $\Sigma$ then there are possibly an infinite number of minimizers for $J$. 
thm 5.4. There exists at least one parameter set $\sigma \in \mathbb{R}^{d}$ such that $\sigma>0$ and that minimizes $J(\sigma)$ in $L^{\infty}$ (defined by eq. (20)). It is given by

$$
\sigma_{j}=H\left(a_{j}\right)+\frac{\delta}{2} \quad(j=1, \ldots, d), \quad \text { where } \delta=\max _{x \in \Sigma}\left(H(x)-\sum_{j=1}^{d} H\left(a_{j}\right) x_{j}\right)
$$

where $H\left(a_{j}\right)=\frac{\sigma_{j}^{(i)} \sigma_{j}^{(e)}}{\sigma_{j}^{(i)}+\sigma_{j}^{(e)}}$ are the harmonic averages of the intra- and extracellular conductivities. It was shown in thm 5.1 that $\delta=0$ iff $H$ is linear or equivalently, $H$ is the symbol of the harmonic average operator and the equal anisotropy ratio assumption holds.

Proof. The existence of $\sigma$ and its value is a corollary of thm. 5.3. Furthermore, $\sigma_{j} \geq \frac{\sigma_{j}^{(i)} \sigma_{j}^{(e)}}{\sigma_{j}^{(i)}+\sigma_{j}^{(e)}}>0$ because $\delta \geq 0$.

\subsubsection{The two-dimensional case}

If the maximizer (from thm. 5.3) of $H_{0}(x)=H(x)-h \cdot x$ is a vertex of $\Sigma$, $x^{\star} \in\left\{a_{1}, \ldots, a_{d}\right\}$, then $\delta=H_{0}\left(x^{\star}\right)=H_{0}\left(a_{j}\right)=0$ and then $H(x)=h \cdot x$ is a linear function or equivalently, $\sigma^{(i)}=k \sigma^{(e)}$. This is independent from the dimension $d$, a fortiori true when $d=2$. We then obtain:

thm 5.5. In the two-dimensional case, $d=2$, the condition $x_{j}^{\star}>0$ for $j=1,2$ is equivalent to $x^{\star} \notin\left\{a_{1}, a_{2}\right\}$ so that, in view of thm. 5.3 and thm. 5.1,

1. either $\sigma^{(i)}=k \sigma^{(e)}$ and $H$ is the symbol of the harmonic monodomain;

2. or $J(\sigma)$ has a unique minimizer defined by eq. (22).

In the latter case, $\sigma^{(i)} \neq k \sigma^{(e)}$, the maximizer $x^{\star}$ is given by

$$
\begin{cases}x_{1}^{\star}=x_{2}^{\star}=\frac{1}{2} & \text { if } \sigma_{1}=\sigma_{2}, \\ x_{1}^{\star}=\frac{\sigma_{2}-\left(\sigma_{1} \sigma_{2}\right)^{1 / 2}}{\sigma_{2}-\sigma_{1}}, x_{2}^{\star}=\frac{\sigma_{1}-\left(\sigma_{1} \sigma_{2}\right)^{1 / 2}}{\sigma_{1}-\sigma_{2}} & \text { if } \sigma_{1} \neq \sigma_{2},\end{cases}
$$

where $\sigma_{1}=\sigma_{1}^{(i)}+\sigma_{1}^{(e)}$ and $\sigma_{2}=\sigma_{2}^{(i)}+\sigma_{2}^{(e)}$.

Proof. The alternative (1) or (2) is a direct consequence of thm. 5.3. Now, in case $(2), x^{\star}$ is a solution of the equation $\nabla H_{0}(x)=0$ because it is a maximizer of $H_{0}$ in the interior of $\Sigma$. With the new parameterization $t=x_{1}$ (and then $\left.x_{2}=1-t\right)$, the functions become

$$
\begin{gathered}
H(t)=\frac{S_{i}(t) S_{e}(t)}{S_{i}(t)+S_{e}(t)} \quad \text { with } S_{i}(t)=\sigma_{1}^{i} t+\sigma_{2}^{i}(1-t), S_{e}(t)=\sigma_{1}^{e} t+\sigma_{2}^{e}(1-t), \\
H_{0}(t)=H(t)-H(1) t-H(0)(1-t) .
\end{gathered}
$$

and $x^{\star}$ is the solution of

$$
H^{\prime}(t)=H(1)-H(0) \text {. }
$$

Consider the notations $a_{i}=\sigma_{2}^{(i)}, b_{i}=\sigma_{1}^{i}-\sigma_{2}^{i}, a_{e}=\sigma_{2}^{(e)}$ and $b_{e}=\sigma_{1}^{e}-\sigma_{2}^{e}$; then $\sigma_{2}=a_{i}+a_{e}$ and $\sigma_{1}-\sigma_{2}=b_{i}+b_{e}$. Note that $\sigma_{2}>0$. The function $H$ reads

$$
\forall t \in[0,1], \quad H(t)=\frac{\left(a_{i}+b_{i} t\right)\left(a_{e}+b_{e} t\right)}{\sigma_{2}+\left(\sigma_{1}-\sigma_{2}\right) t} .
$$

$\mathrm{RR} \mathrm{n}^{\circ} 7810$ 
If $\sigma_{1}=\sigma_{2}$ then easily,

$$
H^{\prime}(t)=\frac{b_{i} a_{e}+b_{e} a_{i}}{\sigma_{2}}+2 \frac{b_{i} b_{e}}{\sigma_{2}} t \quad \text { and } \quad H(1)-H(0)=\frac{b_{i} a_{e}+b_{e} a_{i}}{\sigma_{2}}+\frac{b_{i} b_{e}}{\sigma_{2}}
$$

so that eq. (24) is equivalent to $2 t=1$ and then $t^{\star}=1 / 2$. If $\sigma_{1} \neq \sigma_{2}$, a short computation shows that

$$
\begin{aligned}
H(t)=\frac{a_{i} a_{e}+\left(a_{e} b_{i}+a_{i} b_{e}\right) t+b_{i} b_{e} t^{2}}{\sigma_{2}+\left(\sigma_{1}-\sigma_{2}\right) t} & \\
= & \frac{a_{e} b_{i}+a_{i} b_{e}}{\sigma_{1}-\sigma_{2}}-\frac{\sigma_{2}}{\sigma_{1}-\sigma_{2}} \frac{b_{i} b_{e}}{\sigma_{1}-\sigma_{2}}+\frac{b_{i} b_{e}}{\sigma_{1}-\sigma_{2}} t+\frac{r}{\sigma_{2}+\left(\sigma_{1}-\sigma_{2}\right) t}
\end{aligned}
$$

with $r=a_{i} a_{e}-\sigma_{2}\left(\frac{a_{e} b_{i}+a_{i} b_{e}}{\sigma_{1}-\sigma_{2}}-\frac{\sigma_{2}}{\sigma_{1}-\sigma_{2}} \frac{b_{i} b_{e}}{\sigma_{1}-\sigma_{2}}\right)$. Note that $r \neq 0$, otherwise $H$ is a linear function and $\sigma^{(i)}=k \sigma^{(e)}$. At last equation (24) is equivalent to

$$
\begin{aligned}
& \frac{b_{i} b_{e}}{\sigma_{1}-\sigma_{2}}-\frac{r\left(\sigma_{1}-\sigma_{2}\right)}{\left(\sigma_{2}+\left(\sigma_{1}-\sigma_{2}\right) t\right)^{2}}=\frac{b_{i} b_{e}}{\sigma_{1}-\sigma_{2}}+\frac{r}{\sigma_{1}}-\frac{r}{\sigma_{2}} \\
\Leftrightarrow & \left(\sigma_{2}+\left(\sigma_{1}-\sigma_{2}\right) t\right)^{2}=\sigma_{2} \sigma_{1} \Leftrightarrow t=\frac{1}{\sigma_{1}-\sigma_{2}}\left(-\sigma_{2} \pm \sqrt{\sigma_{2} \sigma_{1}}\right)=\frac{\sigma_{2} \pm \sqrt{\sigma_{2} \sigma_{1}}}{\sigma_{2}-\sigma_{1}} .
\end{aligned}
$$

Now if $\sigma_{1}<\sigma_{2}$ then $0<\sigma_{1}<\sqrt{\sigma_{1} \sigma_{2}}<\sigma_{2}$ and if $\sigma_{2}<\sigma_{1}$ then $0<\sigma_{2}<$ $\sqrt{\sigma_{1} \sigma_{2}}<\sigma_{1}$ so that in any case $0<\frac{\sigma_{2}-\sqrt{\sigma_{1} \sigma_{2}}}{\sigma_{2}-\sigma_{1}}<1$ is the correct solution for $t^{\star}=x_{1}^{\star}$.

\subsubsection{The 3D transversely isotropic case}

In many $3 \mathrm{D}$ situations, the anisotropy of the intra- and extra-cellular conductivities are assumed to be transversely isotropic, meaning that the conductivities in the two directions perpendicular to the fibers are equal. Considering that the first direction is along fibers, it reads:

$$
\sigma_{2}^{(i)}=\sigma_{3}^{(i)}=\sigma_{\perp}^{(i)}, \quad \sigma_{2}^{(e)}=\sigma_{3}^{(e)}=\sigma_{\perp}^{(e)} .
$$

In this situation, the symbols simplify:

$$
\begin{aligned}
& S_{i}(x)=\sigma_{1}^{(i)} x_{1}+\sigma_{\perp}^{(i)}\left(x_{2}+x_{3}\right):=S_{i}\left(x_{1}, x_{\perp}\right), \\
& S_{e}(x)=\sigma_{1}^{(e)} x_{1}+\sigma_{\perp}^{(e)}\left(x_{2}+x_{3}\right):=S_{e}\left(x_{1}, x_{\perp}\right)
\end{aligned}
$$

with $x_{\perp}=x_{2}+x_{3}$ (and then $\left(x_{1}, x_{\perp}\right) \in \Sigma$ ) and

$$
H(x)=\frac{S_{i}\left(x_{1}, x_{\perp}\right) S_{i}\left(x_{1}, x_{\perp}\right)}{S_{i}\left(x_{1}, x_{\perp}\right)+S_{i}\left(x_{1}, x_{\perp}\right)}:=H\left(x_{1}, x_{\perp}\right)
$$

the problem can be solved explicitly using theorem 5.5 for the $2 \mathrm{D}$ case. The $L^{\infty}$ optimal monodomain approximation is given by eq. (22) with

$$
\delta=H\left(x_{1}^{\star}, x_{\perp}^{\star}\right)-\frac{\sigma_{1}^{(i)} \sigma_{1}^{(e)}}{\sigma_{1}^{(i)}+\sigma_{1}^{(e)}} x_{1}-\frac{\sigma_{\perp}^{(i)} \sigma_{\perp}^{(e)}}{\sigma_{\perp}^{(i)}+\sigma_{\perp}^{(e)}} x_{\perp}
$$

and $\left(x_{1}^{\star}, x_{\perp}^{\star}\right)$ given by eq. (23).

rmk 5.5. It was not found any specific analytical expression of the solution in the general, orthotropic, 3D case. However finding a solution from thm 5.4 is always possible through a simple convex optimization algorithm. 


\subsection{The case $p=2$, average quadratic approximation}

Our problem in $L^{2}$ norm is to find $\sigma=\left(\sigma_{1}, \ldots, \sigma_{d}\right) \in \mathbb{R}^{d}$ such that $J(\sigma)=$ $\int_{\mathbb{S}^{d-1}}\left|H(\xi)-S_{\sigma}(\xi)\right|^{2} d \xi$ is minimum over $\mathbb{R}^{d}$.

thm 5.6. There exists a unique parameter set $\sigma \in \mathbb{R}^{d}$ that minimizes $J(\sigma)$. $\sigma$ is the solution of the linear system

$$
M \sigma=b, \quad \text { with } M_{i j}=\int_{\mathbb{S}^{d-1}}\left|\xi_{i}\right|^{2}\left|\xi_{j}\right|^{2} d \xi, \quad b_{i}=\int_{\mathbb{S}^{d-1}} H(\xi)\left|\xi_{i}\right|^{2} d \xi .
$$

Proof. The function $J(\sigma)$ is the Euclidean distance between the $L^{2}\left(\mathbb{S}^{d-1}\right)$ functions $H$ and $S_{\sigma}$ while $E=\left\{S_{\sigma}, \sigma \in \mathbb{R}^{d}\right\}$ is a finite dimensional linear subset of $L^{2}\left(\mathbb{S}^{d-1}\right)$. Hence the problem is solved uniquely by the perpendicular projection of $H$ on the subspace $E$, characterized by

$$
\int_{\mathbb{S}^{d-1}}\left(H(\xi)-S_{\sigma}(\xi)\right) S_{\mu}(\xi) d \xi=0, \quad \forall \mu \in \mathbb{R}^{d} .
$$

Eq. (25) is deduced from this equality by taking successively $\mu=a_{j}$ for $j=$ $1, \ldots, d$, because $S_{a_{j}}(\xi)=\left|\xi_{j}\right|^{2}$.

We were not able to prove the positivity of $\sigma$ when $p=2$.

\section{$6 \quad$ Numerical examples}

Numerical results are presented to illustrate the impact of selecting various monodomain approximations in the two-dimensional case. We first introduce two sets of conductivities. The first one is such that conductivities in the intra- and extra-cellular spaces have ratios that are physiological while the second set have non-physiological ratios giving larger deviation for all monodomain approximations. We next compare the solutions for various monodomain approximations to the solution of the bidomain model using two sets of boundary conditions, first with periodic boundary conditions and second with homogeneous Neumann boundary conditions commonly used for isolated hearts. For 2D computations, the symbols will be represented as functions of the angle $\theta$ that defines the direction of interest: the previous parameter is $\xi=(\cos \theta, \sin \theta) \in \mathbb{S}^{2}$.

\subsection{Comparing monodomain and bidomain symbols}

We first consider conductivities with physiological values (expressed in $\mathrm{mS} / \mathrm{cm}$ ) taken from Ref. [17]. Figure 1 shows a graph of the symbols for various monodomain approximations and the bidomain operator, and a graph of the error between these approximations and the bidomain symbol. The graphs are drawn for $\theta \in[-\pi / 2, \pi / 2]$ but can easily be deduced for all directions given that the monodomain symbol $S$ and bidomain symbol $H$ are both periodic even functions in $\cos ^{2} \theta$. The curve labelled "exact" refers to the bidomain symbol, "Harmonic" to the symbol obtained by using harmonic averages of the conductivities at angles $\theta=0$ and $\theta=-\pi / 2$, "Linf optimal" and "L2 optimal", respectively, to the $L^{\infty}$ and $L^{2}$ optimal monodomain symbols introduced in this paper, and "Nielsen et al" to the symbol of the monodomain operator obtained by setting

$$
\sigma=\frac{k^{\star}}{1+k^{\star}} \sigma^{(i)} \quad \text { where } k^{\star}=\arg \min _{k}\left\|\sigma^{(e)}-k \sigma^{(i)}\right\|_{2} .
$$


This latter monodomain approximation was proposed in Ref. [18] in connection with an optimal control problem to minimize the error between monodomain and bidomain model solutions.

As can be seen on figure 1 at the left, all monodomain symbols are close to the bidomain symbol. The graph of the error at the right shows that the harmonic approximation preserves planar waves moving along the 4 angles $\theta=n \pi / 2$, $n=0,1,2,3$, while the $L^{\infty}$ and $L^{2}$ approximations are exact for eight angles. Consequently, the monodomain approximation (26) is exact for four directions. Assuming that the fibers are aligned along $\theta=0$, wave speed along this direction will only be exact for the harmonic approximation. On the other hand, for directions at $45^{\circ}$ or so with the fibers, none of the monodomain approximations propagate waves with the right speed. The error for the harmonic approximation is twice as large for these waves than for the $L^{\infty}$ approximation. The worst approximation for these angles is given by (26). These facts are confirmed by comparing the $L^{\infty}$ error for these symbols in Table 1. The $L^{\infty}$ and $L^{2}$ errors presented in this table are computed over all $\theta \in[-\pi / 2, \pi / 2]$. As expected from the equioscillation principle, the maximal error is reached at eight points on the unit circle for the $L^{\infty}$-optimal symbol while it is reached at only two or four points for the other three approximations. Finally the $L^{\infty}$ (resp. $L^{2}$ ) error is minimized for the $L^{\infty}$ (resp. $L^{2}$ ) optimal symbol. Of course, the $L^{\infty}$ approximation must be chosen to minimize the plane wave speed error uniformly over all wave numbers.
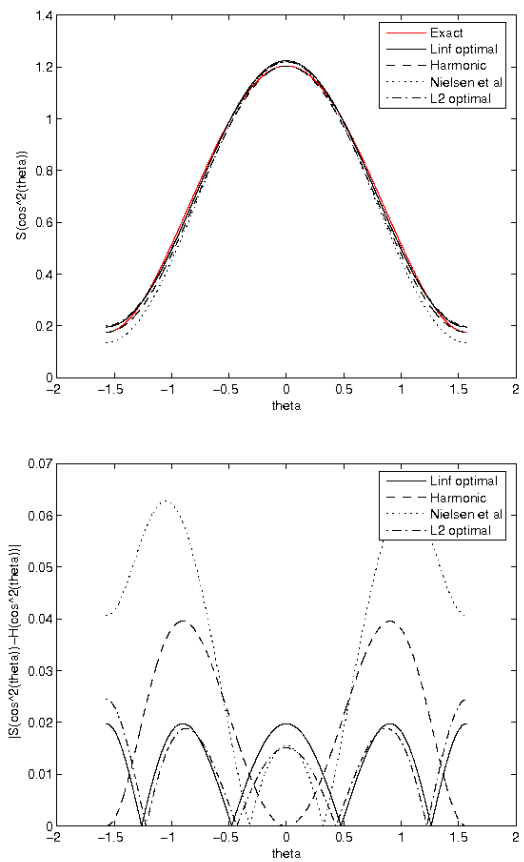

Figure 1: Symbols $S\left(\cos ^{2} \theta\right)$ (left) and error $\left|S\left(\cos ^{2} \theta\right)-H\left(\cos ^{2} \theta\right)\right|$ (right) for various approximate monodomain models. The conductivities $\sigma^{(i)}=$ $[1.741,0.1934]$ and $\sigma^{(e)}=[3.906,1.970]$ are set to common physiological values. 
Table 1: Error between approximate and exact symbols, and computed conductivities for the physiological values of conductivities given in Fig. 1

\begin{tabular}{|c|c|c|c|c|}
\hline Case & $L^{\infty}$ error & $L 2$ error & $\sigma_{1}$ & $\sigma_{2}$ \\
\hline$L^{\infty}$-optimal & 0.01977716 & 0.03505411 & 1.22401764 & 0.19588791 \\
\hline$L 2$-optimal & 0.02443201 & 0.03406934 & 1.21936280 & 0.20054275 \\
\hline Harmonic average & 0.03955433 & 0.06071551 & 1.20424048 & 0.17611075 \\
\hline Nielsen et al & 0.06274502 & 0.10528119 & 1.21979795 & 0.13550196 \\
\hline
\end{tabular}

We next consider non-physiological conductivities. These have been chosen to show a large deviation on the symbols, for instance by taking the largest conductivities in the intra- and extra-cellular media orthogonal to each other. Figure 2 and Table 2 show the graphs of the symbols and the error on the approximate symbols. All the remarks made above in the physiological case apply except for the fact that now the error on the harmonic approximation is as large as on the approximation (26). Comparing errors in Tables 1 and 2 , one notices that the error is about four time larger for non-physiological conductivities than in the physiological case. Both tables give the conductivities $\sigma_{1}$ and $\sigma_{2}$ along and across the fibers, respectively, for the various monodomain approximations.
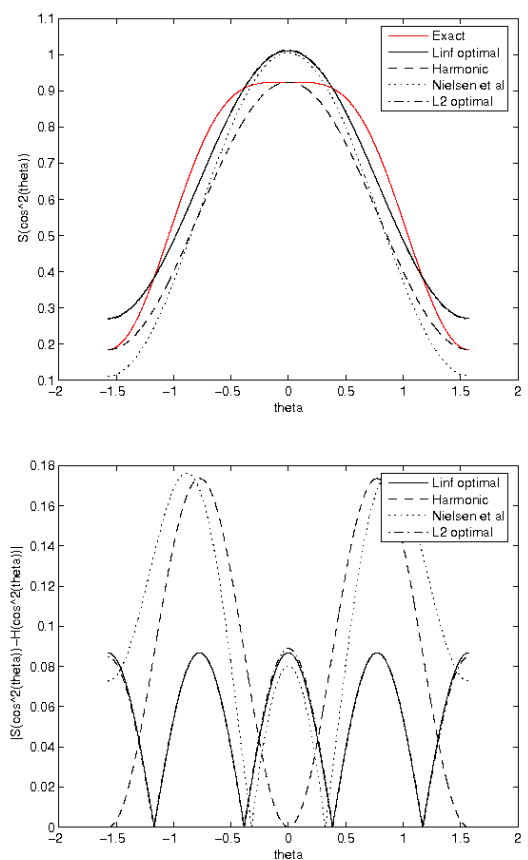

Figure 2: Symbols $S\left(\cos ^{2} \theta\right)$ (left) and error $\left|S\left(\cos ^{2} \theta\right)-H\left(\cos ^{2} \theta\right)\right|$ (right) for various approximate monodomain models. The conductivities $\sigma^{(i)}=$ $[1.741,0.1934]$ and $\sigma^{(e)}=[1.970,3.906]$ are set to non-physiological values. 
Table 2: Error between approximate and exact symbols, and computed conductivities for the non-physiological values of conductivities given in Fig. 2

\begin{tabular}{|c|c|c|c|c|}
\hline Case & $L^{\infty}$ error & $L 2$ error & $\sigma_{1}$ & $\sigma_{2}$ \\
\hline$L^{\infty}$-optimal & 0.08675730 & 0.15377332 & 1.01097449 & 0.27103315 \\
\hline$L 2$-optimal & 0.08891580 & 0.15372572 & 1.01313299 & 0.26887465 \\
\hline Harmonic average & 0.17351460 & 0.26634320 & 0.92421719 & 0.18427584 \\
\hline Nielsen et al & 0.17632642 & 0.29021891 & 1.00451378 & 0.11158699 \\
\hline
\end{tabular}

\subsection{Two-dimensional test cases}

To compare the bidomain model and its various monodomain approximations, we solve (5)-(6) and (1)-(3). The ionic currents are set according to the simple FitzHugh-Nagumo (FHN) model,[13]:

$$
f(u, v)=u(u-a)(u-1)+v \quad \text { and } \quad g(u, v)=\epsilon(\gamma v-u) .
$$

No attempt was made at setting parameters in the FHN model to recover a physiological action potential. Getting maximal accuracy of the numerical solutions was the primary concern. A more elaborate ionic model can be used but the conclusions should remain the same.

The models are discretized in space using finite element methods on unstructured triangular meshes and in time using second order Backward Differentiation Formula (BDF2). The test cases with homogeneous Neumann boundary conditions were solved with quadratic finite elements and the fully implicit BDF2 scheme using $\mathrm{MEF}++,[14]$ while periodic boundary conditions called for the use of another code, Freefem,$++[15]$ where these boundary conditions were already implemented. Linear elements and a semi-implicit BDF2 scheme with a coupled solution of the linear system involving Eq. (1) and (3) were implemented in FreeFEM++. These two time-stepping schemes were analyzed and shown to be second order accurate in Ref. [12]. The independence of the numerical solutions with respect to mesh size and time step was verified by refining the mesh and decreasing the time step up to the point where the solutions look identical on successive meshes.

\subsubsection{Periodic boundary conditions}

Planar wave propagation with an arbitrary angle with respect to fibers makes sense in the whole space $\mathbb{R}^{d}$. Usual Neumann or Dirichlet boundary conditions guide waves along the domain boundary, leading to propagating planar waves that quickly degenerate into non-planar waves. To circumvent this issue, we used periodic boundary conditions on rectangles and planar waves propagating parallel to one side of the rectangle. This trick allows fibers with an arbitrary constant angle. The domain chosen is $[0,200] \times[0,10]$ and is discretized with an unstructured triangular mesh with 800 and 40 elements along the $x$ and $y$ axis, respectively. The time step was set to $\Delta t=0.2$ time unit for all results presented. The action potential waves are initiated with the following initial solution:

$$
u(x, 0)=H(20-x) H(x-10) \quad \text { and } \quad v(x, 0)=0.1 H(12-x) H(x-8),
$$


where $H=H(x)$ is the Heaviside function. The extra-cellular potential $u_{e}$ is initially set to zero for the bidomain model. This initial condition generates a single wave propagating in the positive $x$-direction. The parameters in the FHN model are chosen as $a=0.139, \epsilon=0.002, \gamma=2.54$.

Figure 3 shows the time-evolution of the trans-membrane potential $u$ from initial time to the final time $T=460$ at which the solutions are compared. The action potential is initiated with a square wave at the left of the domain, propagates to the right to reach the rightmost boundary and reenter the domain at the left from the periodicity conditions. Figure 4 compares the solutions obtained from the harmonic and $L^{\infty}$ monodomain approximations and the bidomain model in three cases, namely with physiological conductivities and fibers at 0 and 45 degrees (resp. Fig.(a) and (b)), and with non-physiological conductivities and fibers at 45 degrees (Fig.(c)). As expected from the analysis of the symbols presented above, the graphs of the harmonic monodomain and bidomain solutions are superposed at 0 degree. The wave for the $L^{\infty}$ monodomain is ahead of the bidomain solution, which is also consistent with a larger symbol for this approximation than the bidomain symbol at 0 degree. At 45 degrees, both in the physiological and non-physiological cases, the monodomain solutions lag behind the bidomain solution with the largest gap for the harmonic approximation. This is consistent with the fact that monodomain symbols are smaller than the bidomain symbol at this angle. Deviations of the monodomain solutions from the bidomain solution are greater in the non-physiological case, consistently with larger symbol errors in this case.

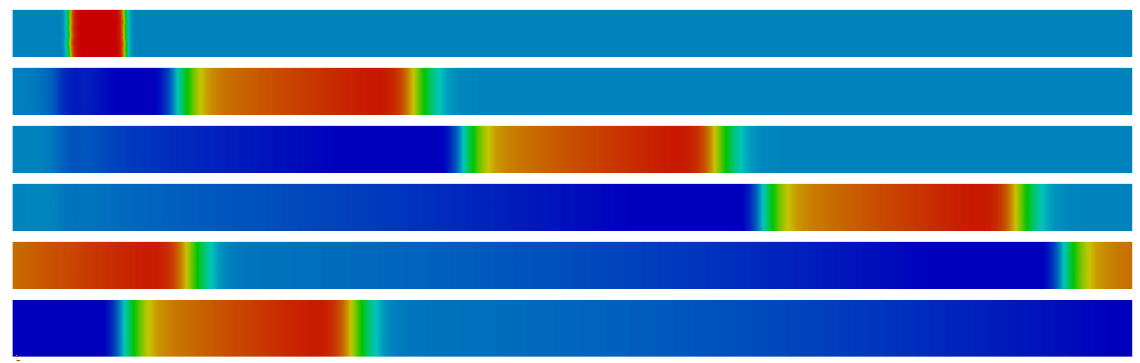

Figure 3: Trans-membrane potential at times $T=0,100,200,300,400,460$ (from top to bottom) for the bidomain model with physiological conductivities and fibers at 0 degree

We quantified the differences between solutions by computing propagation speed in Table 3. Of course, the propagation speed computed are consistent with the graphs shown on Figure 4, in the sense that if a monodomain solution lags behind the bidomain solution the corresponding speed is smaller, and viceversa. At 0 degree, the propagation speed for the harmonic approximation must be exact but the absolute error on this speed is about $10^{-5}$ due to numerical accuracy of the finite element method. This strengthens our confidence in the proper numerical resolution of the models. In table 3 , we computed the relative error on the speed as $\frac{c_{m}-c_{b}}{c_{b}}$, where $c_{b}$ and $c_{m}$ are the speed of the bidomain and monodomain potential wave, respectively, for any of the monodomain approximations. From the relation $c(\theta)=k \sqrt{S(\theta)}$ between the wave velocity $c=c(\theta)$ 


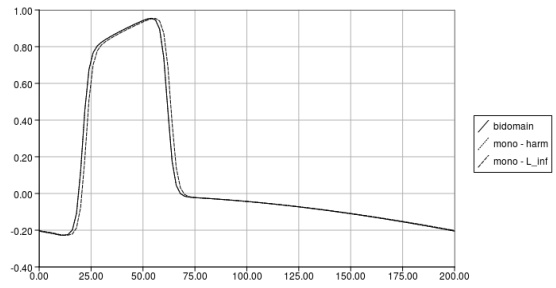

(a)

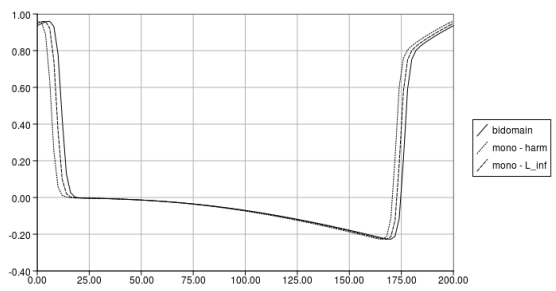

(b)

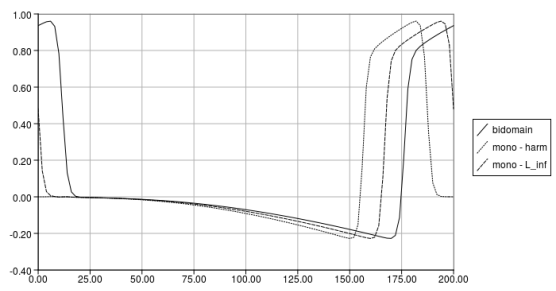

(c)

Figure 4: Plot of the trans-membrane potential along a horizontal cut at time $T=460$ : (a) Physiological conductivities, fibers at 0 degree; (b) Physiological conductivities, fibers at 45 degrees; (c) Non-physiological conductivities, fibers at 45 degrees.

in direction $\theta$ and the conductivity or symbol $S=S(\theta),[6]$ we deduce that

$$
\frac{c_{m}-c_{b}}{c_{b}}=\frac{\sqrt{S(\theta)}}{\sqrt{H(\theta)}}-1,
$$

where as before $S(\theta)$ and $H(\theta)$ are the symbols of the monodomain and bidomain operators, respectively, taken in the direction $\theta$. The values of the expression on the right-hand-side are given in Table 4 . As can be observed by comparing the errors reported in Tables 3 and 4, the theory on approximate symbols proposed here accurately predicts the differences on propagation velocities for the monodomain approximations, at least for the planar waves with periodic boundary conditions computed in this section.

Table 3: Speed of the potential wave for the solutions shown on Fig. 4 (relative error on the speeds are given in parenthesis)

\begin{tabular}{|l|l|l|l|}
\hline Case & Bidomain & Harmonic average & $L^{\infty}$-optimal \\
\hline Physiological - $0^{\circ}$ & 0.537335 & $0.537326(0.00 \%)$ & $0.541574(0.79 \%)$ \\
\hline Physiological - $45^{\circ}$ & 0.417956 & $0.406847(-2.66 \%)$ & $0.412732(-1.25 \%)$ \\
\hline Non-physiological - 45 & 0.417754 & $0.364145(-12.83 \%)$ & $0.391479(-6.29 \%)$ \\
\hline
\end{tabular}

\subsubsection{Homogeneous Neumann boundary conditions}

For our second test case, we consider a spherical wave propagating on the square $[-30,30] \times[-30,30]$ subject to homogeneous Neumann boundary conditions. The domain is discretized with a regular mesh of $200 \times 200$ squares divided into 
Table 4: Relative error on the square root of symbols for the solutions shown on Fig. 4

\begin{tabular}{|l|l|l|}
\hline Case & Harmonic average & $L^{\infty}$-optimal \\
\hline Physiological $-0^{\circ}$ & $0.00 \%$ & $0.82 \%$ \\
\hline Physiological $-45^{\circ}$ & $-2.61 \%$ & $-1.22 \%$ \\
\hline Non-physiological $-45^{\circ}$ & $-12.73 \%$ & $-6.14 \%$ \\
\hline
\end{tabular}

two triangles each. We used quadratic finite elements, which amounts to a grid of $401 \times 401$ in terms of number of degrees of freedom. The time step was set to $\Delta t=0.5$ time unit for all results presented. The action potential waves are initiated with the following initial solution:

$$
u(x, 0)=1 \text { for } x^{2}+y^{2}<25,0 \text { elsewhere, and } v(x, 0)=0 .
$$

For the bidomain model, the extra-cellular potential $u_{e}$ is initially set to:

$$
u_{e}(x, 0)=-0.5 \text { for } x^{2}+y^{2}<25,0 \text { elsewhere. }
$$

This initial condition generates a target wave propagating away from the center of the square. The parameters in the FHN model are chosen as $a=0.25$, $\epsilon=0.0016875, \gamma=5.926$.

Figure 5 shows the time-evolution of the trans-membrane potential $u$. The wave is initiated as a spherical wave but becomes elliptical due to the different conductivities in the $x$ - and $y$-directions. When the wave reaches the boundary of the domain, contours are orthogonal to the boundary and propagation occurs along the boundary. The depolarization and repolarization isochrons shown on Figure 6 are obtained by recording at each point of the domain the time at which the trans-membrane potential $u$ crosses the value 0.5 from below and above, respectively. On should note how the target pattern quickly evolves in two nearly planar waves radiating vertically away from the square mid-line.

A plot of the depolarization isochrons on a quarter of the square (of course with solutions computed on the whole square) is used to compare various monodomain approximations. Figure 7 shows these isochrons. As expected, the non-physiological case leads to larger deviations between the bidomain and monodomain isochrons, and the error grows with time. The error is dependent upon the propagation angle. Minimal propagation error is seen along the $x$ - and $y$ axis for the harmonic approximation, at least up to the point where the wave is close enough to the boundary and propagation is spoiled by the Neumann boundary conditions. For the $L^{\infty}$-optimal approximation, minimimal propagation error is observed along an oblique at about thirty to fourty degrees with the $x$-axis. Because the domain is small, propagation along the domain boundary quickly dominates and selecting the optimal monodomain approximation is not as critical. In fact, the wave approaching a planar wave moving vertically and the harmonic approximation being exact along this direction, it gives a better solution. The isochrons of the $L^{2}$-optimal solution are not shown but are very close to those of the $L^{\infty}$ solution. 


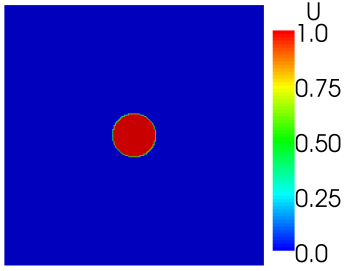

$T=0$

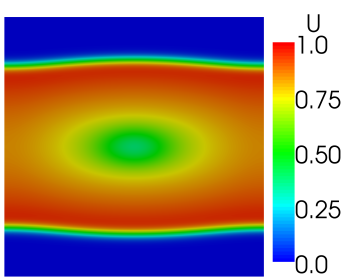

$T=150$

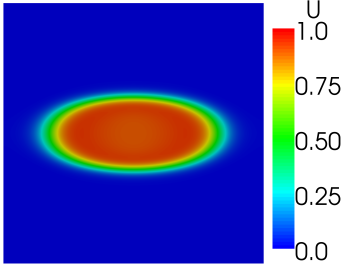

$T=50$

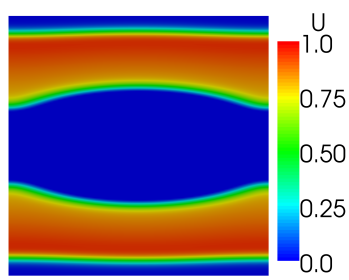

$T=200$

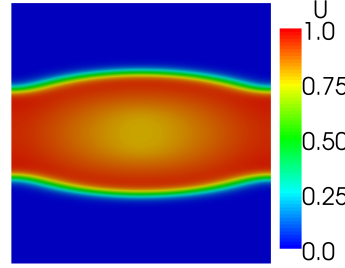

$T=100$

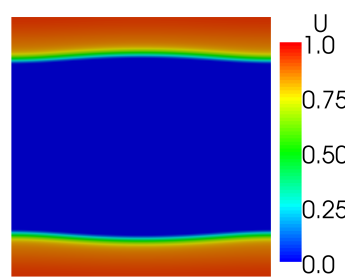

$T=250$

Figure 5: Trans-membrane potential at different times for the bidomain model with physiological conductivities

\section{Acknowledgments}

The authors would like to thank the Laboratoire Jean Leray, Université de Nantes, for supporting stay of the first and second authors in Nantes. This work was also supported by a NSERC Discovery Grant to the first author, a NSERC Graduate Scholarship to the second author and an ANR Grant (JCJC04-0141) to the third author. The authors wish to thank Nicolas Depauw from Université de Nantes for fruitful discussions.

\section{References}

[1] R. Aliev and A. Panfilov. A simple two-variable model of cardiac excitation. Chaos, Solitons and Fractals, 3(7):293-301, 1996.

[2] S. Alinhac and P. Gérard. Opérateurs pseudo-différentiels et théorème de Nash-Moser. Savoirs Actuels. InterEditions/Editions du CNRS, 1991.

[3] M. Bendahmane and K. H. Karlsen. Analysis of a class of degenerate reaction-diffusion systems and the bidomain model of cardiac tissue. Netw. Heterog. Media, 1(1):185-218 (electronic), 2006.

[4] M. Boulakia, M.A. Fernandez, J.-F. Gerbeau, and N. Zemzemi. A coupled system of pdes and odes arising in electrocardiograms modeling. AMRX. Appl. Math. Res. Express, 2008, 2008.

[5] Y. Bourgault, Y. Coudière, and C. Pierre. Existence and uniqueness of the solution for the bidomain model used in cardiac electrophysiology. Nonlinear Anal. Real World Appl., 10(1):458-482, 2009. 

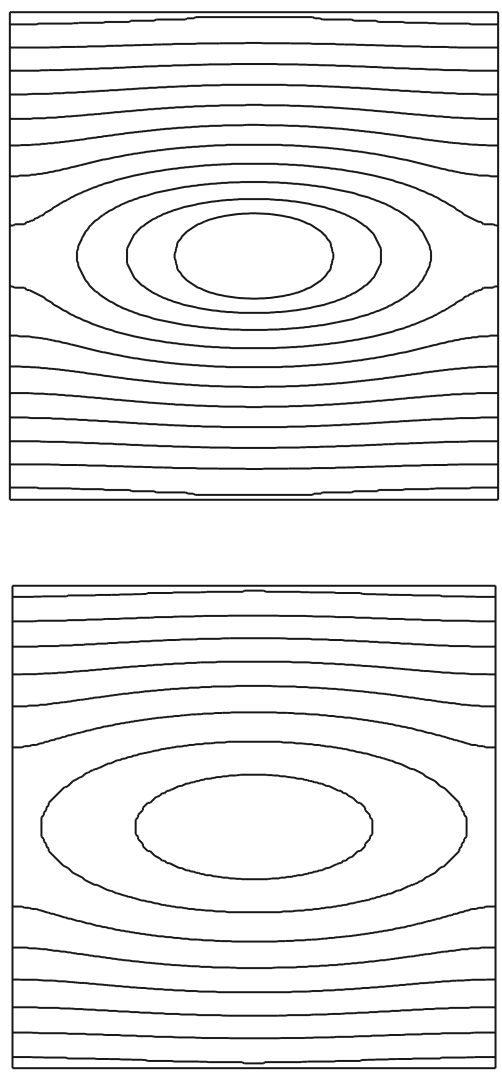

Figure 6: Isochrons of depolarization (left) and repolarization (right). The time interval between two isochrons is 20 time units. 

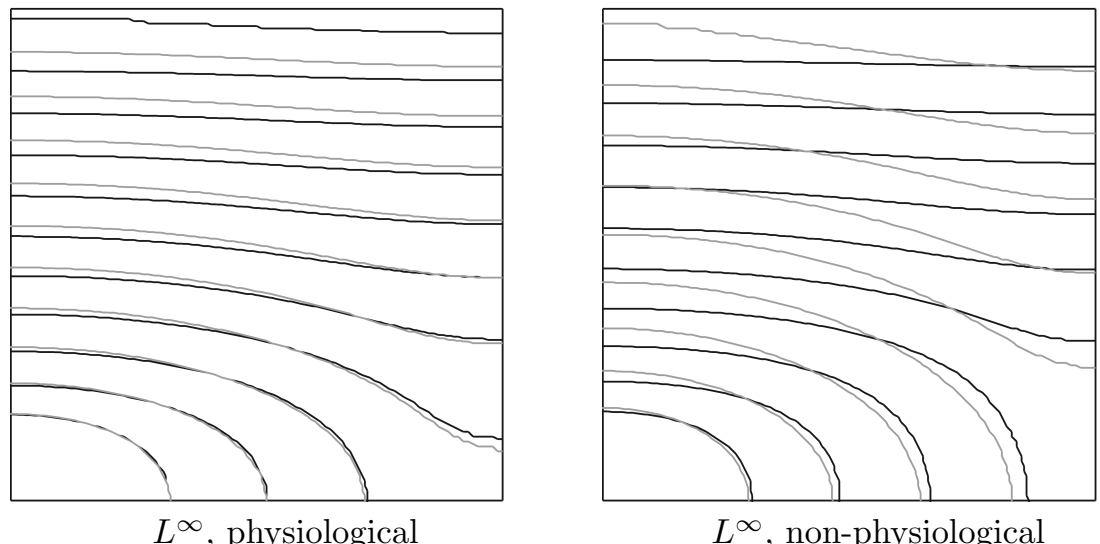

$L^{\infty}$, non-physiological

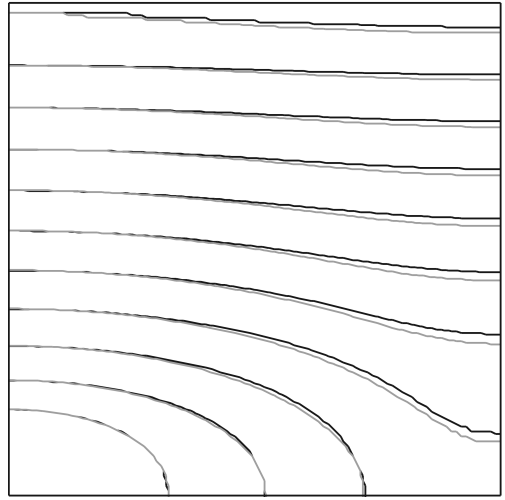

harmonic, physiological

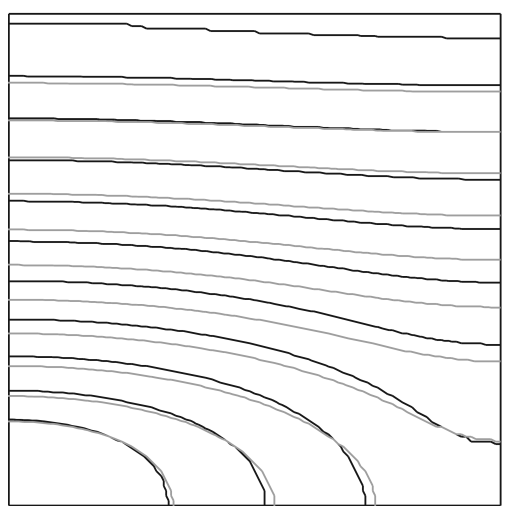

Nielsen et al, physiological

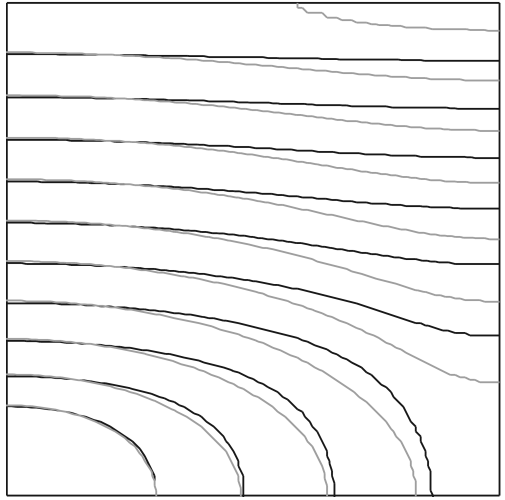

harmonic, non-physiological

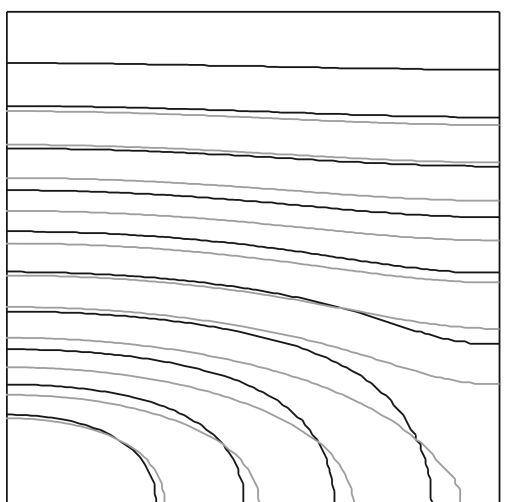

Nielsen et al, non-physiological

Figure 7: Comparison of depolarization isochrons for the bidomain model (dark lines) versus various monodomain model approximations (gray lines). The upper right quadrant of the domain is shown. 
[6] N.F. Britton. Reaction-diffusion equations and their applications to biology. London etc.: Academic Press, 1986.

[7] D.G. Schaeffer C.C. Mitchell. A two-current model for the dynamics of cardiac membrane. Bulletin Math Bio, 65:767-793, 2003.

[8] P. Colli Franzone, L. Guerri, and S. Tentoni. Mathematical modeling of the excitation process in myocardial tissue: Influence of fiber rotation on wavefront propagation and potential field. Math. Biosci., 101(2):155-235, 1990.

[9] P. Colli Franzone, L.F. Pavarino, and B. Taccardi. Simulating patterns of excitation, repolarization and action potential duration with cardiac bidomain and monodomain models. Mathematical Biosciences, 197(1):35-66, September 2005.

[10] Piero Colli Franzone and Giuseppe Savaré. Degenerate evolution systems modeling the cardiac electric field at micro- and macroscopic level. In Evolution equations, semigroups and functional analysis (Milano, 2000), volume 50 of Progr. Nonlinear Differential Equations Appl., pages 49-78. Birkhäuser, Basel, 2002.

[11] M. Crouzeit and A. L. Mignot. Analyse Numérique Des Équations Différentielles. Masson, 1984.

[12] Marc Ethier and Yves Bourgault. Semi-implicit time-discretization schemes for the bidomain model. SIAM J. Numer. Anal., 46(5):2443-2468, 2008.

[13] R.A. FitzHugh. Impulses and physiological states in theoretical models of nerve membran. Biophys. J., 1:445-466, 1961.

[14] GIREF. http://www.giref.ulaval.ca/projets/.

[15] F. Hecht. Freefem++, 3rd edition. Technical Report version 3.7-1, Laboratoire Jacques-Louis Lions, Université Pierre et Marie Curie, Paris.

[16] J. Keener and J. Sneyd. Mathematical Physiology. Springer, 2004.

[17] P. Le Guyader, F. Trelles, and P. Savard. Extracellular measurement of anisotropic bidomain myocardial conductivities. I. theoretical analysis. $A n$ nals Biomed. Eng., 29(10):862-877, 2001.

[18] Björn Fredrik Nielsen, Tomas Syrstad Ruud, Glenn Terje Lines, and Aslak Tveito. Optimal monodomain approximations of the bidomain equations. Appl. Math. Comput., 184(2):276-290, 2007.

[19] Charles Pierre and Yves Bourgault. A comparison of the bidomain and the adapted monodomain models in electro-cardiology. Technical report, December 2010.

[20] M. Potse, B. Dube, J. Richer, A. Vinet, and R. M Gulrajani. A comparison of monodomain and bidomain Reaction-Diffusion models for action potential propagation in the human heart. IEEE Transactions on Biomedical Engineering, 53(12):2425-2435, December 2006. 
[21] J.M. Roger and A.D. McCulloch. A collocation-Galerkin finite element model of cardiac action potential propagation. IEEE Trans. Biomed. Engr., 41:743-757, 1994.

[22] J. Smoller. Shock Waves and Reaction-Diffusion Equations, volume 258 of Grundlehren der mathematischen Wissenschaften. Springer, 2nd ed., 1994. ISBN: 978-0-387-94259-9.

[23] J. Sundnes, G. T. Lines, X. Cai, B. F. Nielsen, K.-A. Mardal, and A. Tveito. Computing the Electrical Activity in the Heart, volume 1 of Monogr. Comput. Sci. Eng. Springer, September 2006.

[24] Joakim Sundnes, BjÃ,rn Fredrik Nielsen, Kent Andre Mardal, Xing Cai, Glenn Terje Lines, and Aslak Tveito. On the computational complexity of the bidomain and the monodomain models of electrophysiology. Annals of Biomedical Engineering, 34:1088-1097, May 2006.

[25] Marco Veneroni. Reaction-diffusion systems for the macroscopic bidomain model of the cardiac electric field. Nonlinear Anal. Real World Appl., 10(2):849-868, 2009. 


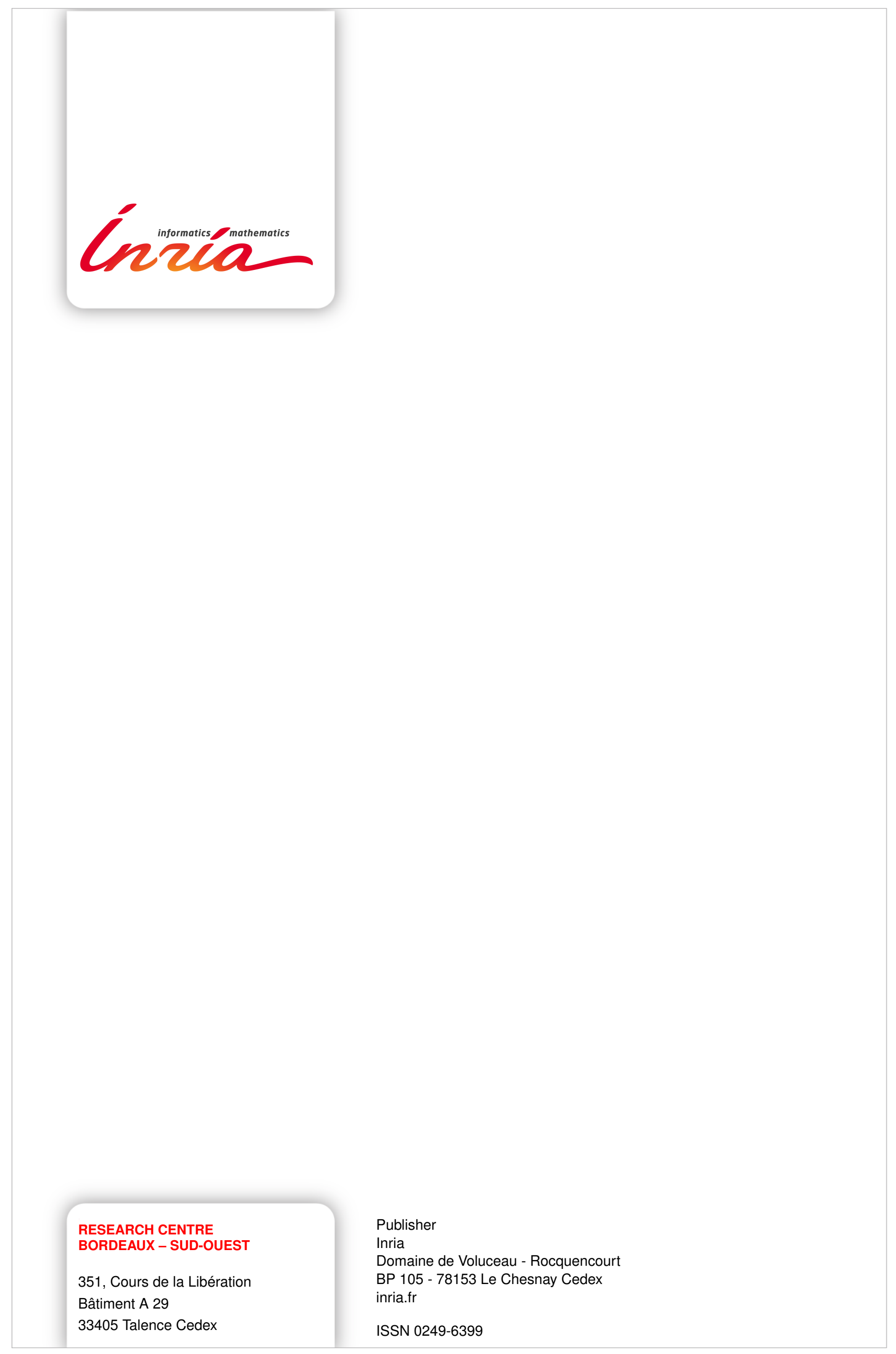

